\title{
Perturbation Theories of the Thermodynamics of Polar and Associating Liquids: a Historical Perspective
}

\author{
Keith E. Gubbins \\ North Carolina State University, Department of Chemical \& Bimolecular Engineering, Raleigh, \\ NC 27695-7905, U.S.A.
}

\begin{abstract}
A historical account is given of the development of perturbation theories for polar and associating liquids. A perturbation expansion of the free energy of a polar liquid about that for a liquid of spherical, non-polar molecules was first proposed by J.A. Barker in 1951, but its further development had to await the successful development of theories for simple, non-polar molecules, which did not occur until the late 1960's. The development of such approaches was further catalyzed by the first molecular simulation results for such polar liquids in the early 1970's. Although the Barker expansion did not converge well for strong electrostatic forces, a resummation using a Padé approximant due to G. Stell and coworkers gave excellent results for the free energy and other thermodynamic properties. A further major advance was the theory proposed by M.S. Wertheim in the mid-1980's, which accounted for the effects of highly directional forces that result in molecular association (H-bonding, charge transfer, etc.). Over the past 30 years this theory has been extended and found to give very good results for a wide variety of applications, including mixtures containing strongly associating molecules such as water, polymers, proteins, and other complex molecules.
\end{abstract}

\section{Introduction}

The search for a satisfactory theory of simple liquids of spherical, nonpolar molecules, such as hard spheres or Lennard-Jones molecules, occupied much effort in the first part of the $20^{\text {th }}$ century. In particular, the period 1953 to 1970 saw the introduction of integral equation theories and perturbation theories that were finally successful for such fluids, first for hard spheres and subsequently for liquids of simple molecules with attractive forces, such as Lennard-Jones and square-well fluids. These developments were much catalyzed by the appearance of the first 
molecular simulations of simple liquids towards the end of this period, providing the means to unambiguously test the theories, and giving crucial guidance to their improvement.

The development of soundly based theories that could be usefully applied to polar and associating liquids had to await these developments, so that the history of theories for such liquids is rather short, dating from about 1951. These theories include integral equation theories (approximate closures that enabled solutions of the Ornstein-Zernike equation or its site-site form, including Percus-Yevick, hypernetted-chain, mean spherical approximation, reference interaction site model, and their extensions), perturbation theories of various kinds and the fluctuation theory of mixtures. In what follows, the development of these theories will be outlined, and their merits and limitations discussed. The discussion of integral equation theories will be brief, since, while useful for predicting liquid structure, so far they have proved less accurate for thermodynamic properties. As concluded by Gray and Gubbins [1] in a survey of perturbation and integral equation theories for fluids of non-spherical molecules in 1984:

The elementary perturbation theory...has a definite advantage in its simplicity and accuracy for thermodynamic properties.

In this essay I will focus on theories that are firmly based in statistical mechanics, i.e. are based on a well-defined Hamiltonian and proceed by well-defined approximations. Such theories possess two distinct advantages over more empirical approaches. First, they can be tested against molecular simulation results, in which the intermolecular force laws and other modeling approximations are the same as those in the theory, thus eliminating major uncertainties inherent in direct comparison with experimental data, where assumptions concerning the force laws are required in addition to approximations in the theory. In the period up to the late 1960's, before the advent of molecular simulations, comparison with experimental data was the only method available, and the fitting of various parameters obscured defects in the theory. The great majority of these theories were found to fail once simulations for simple liquids became available. The second reason for restricting consideration to theories based on a well-defined molecular model is that they can usually be improved by refining the approximations made.

\section{Background}


The history of the theory for liquids of simple spherical molecules has been extensively reviewed [e.g. 2-8], and we give here only a few highlights needed to understand the later development of theories of polar and associating liquids. Of particular importance for these later advances was the development of accurate theories for fluids of hard spheres and of LennardJones molecules, the latter being the prototype for spherical molecules with attractive dispersion forces. These advances were made in the period 1953 to 1971.

The publication of J. Willard Gibbs' treatise on statistical mechanics in 1902 [9], with its clear treatment of the canonical, microcanonical and grand ensembles, opened the way to a more modern treatment of fluids, as was recognized by Hendrik Lorentz working at Leiden University, among others. Lorentz's student, Leonard Salomon Ornstein, in his dissertation [10] developed Gibbs's formal results into a more useful form, and applied them to the study of density fluctuations, later collaborating with Frits Zernike at Groningen University on this topic [11]. This work resulted in the Ornstein-Zernike equation for the total correlation function, $h(r)=g(r) \quad 1$, where $g(r)$ is the familiar radial distribution function. In modern notation this equation is

$$
h\left(r_{12}\right)=c\left(r_{12}\right)+\quad d \mathbf{r}_{3} c\left(r_{13}\right) h\left(r_{23}\right)
$$

where $=N / V$ is number density and $c(r)$ is the direct correlation function; for dense fluids $c(r)$ is short-ranged compared to $g(r)$. Ornstein and Zernike were primarily interested in applying this equation to the study of density fluctuations near the critical point, as had been observed by Thomas Andrews. It was almost four decades later that the Ornstein-Zernike (OZ) equation was first used [12] as the starting point for several important integral equation theories. The $\mathrm{OZ}$ equation serves as a definition of $c(r)$, but a 'closure relation', $c=c[h]$, is needed to obtain a solution for $h$ and hence $g$. The latter is an approximation, and for fluids of simple, spherical molecules two such closures were proposed in the late 1950's, the hypernetted chain (HNC) [13] and Percus-Yevick (PY) [14] approximations:

$$
\begin{array}{llll}
h & c=\ln y & & (\mathrm{HNC}) \\
h & c=y & 1 & (\mathrm{PY})
\end{array}
$$


where $y(r)=g(r) \exp [u(r) / k T]$ is the indirect correlation function (note that for a dilute gas $g(r)=c(r)=\exp [u(r) / k T]$ so that $y=1$; thus $(y-1)$ gives a measure of the indirect correlation for a dense fluid).

Although a fluid of hard spheres does not occur in nature, it's properties are simpler than those of real fluids in which attractive forces are present, and it plays an important role as a reference system in theories of polar and associating liquids. For a hard sphere system the compressibility factor, $p V / N k T$, and the direct and total correlation functions depend only on the density, and not on the temperature. Moreover, there is no gas-liquid transition, the only transition for the pure hard sphere system being the fluid-solid one. Much effort was spent on understanding the behavior of such a fluid in the late 1950's and 1960 's, and theory and molecular simulation provided complementary routes to such developments.

For a fluid of hard spheres of diameter $\sigma$ the PY theory has a simple analytic solution for the direct correlation function, and hence for the radial distribution function from eqn. (2), as was shown independently by Wertheim [15] and by Thiele [16] in 1963. When this solution is used in the pressure equation and the compressibility equation [1] two somewhat different equations of state are obtained for the pressure of the hard sphere fluid:

$$
\begin{gathered}
\frac{p V}{N k T}=\frac{1+2+3^{2}}{(1 \quad)^{2}} \quad \text { (pressure route) } \\
\frac{p V}{N k T}=\frac{1++^{2}}{(1 \quad)^{3}} \quad \text { (compressibility route) }
\end{gathered}
$$

where $=(16) \quad{ }^{3}$ is the packing fraction, i.e. the fraction of space occupied by the hard spheres. From molecular simulation results, for a close-packed crystal $\eta=0.74$, while for a random close packed hard sphere glass $\eta \sim 0.64$. The fluid-solid transition occurs at a fluid packing fraction of $\eta \sim 0.49$. The PY solution for hard spheres applies only to the fluid phase, the theory giving unphysical results in the solid region.

The first molecular simulation studies of fluids were developed in the late 1940s and early 50's in U.S. government laboratories, and grew out of the Manhattan Project and the need for statistical sampling methods to study the diffusion and scattering of neutrons. 
While several researchers were involved in developing the Monte Carlo method $[17,18,19]$, the first clear account of the method was given in 1953 by Nicholas Metropolis and colleagues, working at Los Alamos National Laboratory, who used it to study a fluid of hard discs in two dimensions [20], and soon after for a three-dimensional hard sphere fluid [21]. Subsequently, Berni Alder and Tom Wainwright, working at the Lawrence Radiation Laboratory at Livermore (later renamed Lawrence Livermore National Laboratory) developed the molecular dynamics method, in which the classical equations of motion were solved for a system of $N$ molecules in the microcanonical ensemble. The method and its application to study the fluid-solid phase transition for hard spheres, was first presented at a meeting in Brussels in 1956 [22] and published in a Letter to the Editor one year later in Journal of Chemical Physics [23]. A more detailed account was given two years later [24]. In the course of this work it was shown that a fluid to solid phase transition occurs in the hard sphere system at a reduced density of $\eta \sim 0.46$; such a transition had been predicted earlier by Kirkwood. A good account of the early history of molecular simulation and of this early work on simple fluids has been given by Wood [25].

These early molecular simulation studies and subsequent ones for larger systems meant that ample 'experimental' equation of state data for the hard sphere system was available by 1963 for testing the HNC theory and the PY results of eqns. (3) and (4). When the compressibility factor, $p V / N k T$, is plotted vs. packing fraction, $\eta$, eqns. (3) and (4) give different curves, the compressibility-route curve lying above and the pressure-route curve below the simulation results for the fluid branch, with the compressibility-route giving the more accurate result. The difference between the results from the two routes indicate inconsistency resulting from the PY approximation. The HNC theory also showed such inconsistency, but gave significantly poorer agreement with the MD results than did the PY theory. Despite these inconsistencies, the results of this test of the PY theory showed that it was superior to previous treatments of the hard sphere fluid. If one takes one third of the pressure route PY result and two thirds of the compressibility route result one obtains a result that is indistinguishable from the molecular simulation results, within the error bars of the simulation data. This is the Carnahan-Starling equation [26], 


$$
\frac{p V}{N k T}=\frac{1++^{2} \quad 3}{(1 \quad)^{3}}
$$

although Carnahan and Starling arrived at this equation by a different argument, based on an examination of the hard sphere virial coefficients. The Carnahan-Starling equation was extended to mixtures by Boublik [27], and his equation was later shown to be in good agreement with molecular simulation data for hard sphere mixtures by Mansoori et al. [28], provided that the differences in molecular diameter are not very large.

For liquids composed of molecules with attractive dispersion forces, in addition to repulsive forces, the integral equation approaches have given results for thermodynamic properties that are in only fair agreement with molecular simulation and experiment [29], with the PY theory giving, in general, better results than the HNC theory [30].

Perturbation theories have proved more successful for thermodynamics of such fluids, and were actively pursued in the period 1951 to 1971 . The first such application to liquids was made in 1951 by Christopher Longuet-Higgins [31], working at the University of Manchester. He considered a mixture of components that were conformal, i.e. whose intermolecular potentials conformed to the same functional form, $u(r)=f(r /)$, differing only in the values of the potential parameters, $\varepsilon_{\alpha \beta}$ and $\sigma_{\alpha \beta}$. By expanding the free energy in powers of the differences in these parameters for different components, he was able to relate the real mixture to an ideal one, in which the components remained distinguishable but all had the same potential parameters. When molecular simulation results became available in 1968 it was shown that his expansion converged poorly, but this was found to be due to a poor choice of expansion parameters. Longuet-Higgins expanded in powers of $\left(\varepsilon_{\alpha \beta}-\varepsilon_{X}\right)$ and $\left(\sigma_{\alpha \beta}-\sigma_{X}\right)$, where $\varepsilon_{X}$ and $\sigma_{X}$ are the corresponding parameters for the reference fluid. In spite of its limitations, the theory of Longuet-Higgins was seminal in providing a new route to a theory of liquids, free of the constraints of lattice theories, which were much in vogue at the time. His theory was followed by other similar attempts (see refs. 2 to 8 for details), most of which also gave poor results when later tested against molecular simulations, with the exception of mixtures in which the molecules were all of nearly the same size. However, in 1962 Thomas Leland, working with Patsy Chappelear and Bernard Gamson at Rice University, proposed a theory [32] that 
overcame this problem, by using as expansion parameters ${ }^{3}$ and ${ }^{3}$. These expansion parameters are suggested by examination of the statistical mechanical equations for the pressure and internal energy. The theory leads to the mixing rules:

${ }_{x}=\frac{x x^{3}}{x x^{3}}$
${ }_{x}^{3}=x^{3}$

where $x$ is the mole fraction of component $\alpha$. When molecular simulations for LennardJones liquid mixtures later became available, it was found that this theory worked remarkably well $[3,4,7,8]$, giving good results even for well depth ratios as high as 2 , and molecular volume ratios as high as 25 [33]. Leland later spent a sabbatical leave with John Rowlinson at Imperial College London, and during this period it was realized [34] that the mixing rules of eqn. (6) were essentially the same as those proposed by van der Waals in his 1890 paper [35] on mixtures. As a result the theory is now referred to as the 1-fluid van der Waals theory of mixtures ('1-fluid' because the free energy of the mixture is mapped onto that of a single, conformal reference fluid; other versions of conformal solution theory, termed two-fluid theories, had expanded about an ideal mixture of two different reference fluids, but these theories were later shown to be in poorer agreement with simulations than the one-fluid theory).

An alternative perturbation approach is to expand the free energy of the liquid of interest about that for a fluid in which the molecules interact only with repulsive forces, the attractive forces serving as the perturbation. The first such attempt was by Robert Zwanzig, working at Yale University, in 1954 [36], who took the reference system to be a fluid of hard spheres of diameter $d$ with pair potential $u_{d}=\infty$ for $r \leq d, u_{d}=0$ for $r>d$. This gave good results for high temperature gases, but failed at temperatures in the liquid range. The use of a hard sphere fluid as reference means that the perturbing potential becomes infinite and negative inside the hard core. Later attempts used as reference a fluid whose molecules interacted with a soft repulsive potential. The free energy of such a fluid can be related to that of a hard sphere fluid through a further perturbation expansion [37]. It was John Barker, working at C.S.I.R.O. in Melbourne, and Douglas Henderson at the University of Waterloo who developed the first successful theory of this type in 1967. Much of the work 
was done while Henderson was on sabbatical leave with Barker in Melbourne in the period 1966-67. A preliminary report of their work [38] was given at the Discussion of the Faraday Society on The Structure and Properties of Liquids, held in Exeter in April, 1967, and a more complete account was published later that year [39]. Barker and Henderson (BH) took the potential to be the Lennard-Jones (LJ) model, and the reference fluid to be one in which the molecules interacted with the repulsive part of the LJ potential for $r \leq \sigma$, and 0 for larger separations. The properties of this repulsive reference fluid were further related to those of a hard sphere fluid through a second perturbation expansion. This results in a hard sphere diameter, $d$, that is temperature-dependent, $d$ becoming smaller as temperature increases. Their theory gave quite good results at first order for dense liquids, but required an estimate of the second order term for quantitative accuracy. Even better results for dense liquids were obtained by Weeks, Chandler and Andersen (WCA) [40], who took the reference potential to be the repulsive part of the LJ potential down to the minimum of the potential well, resulting in rapid convergence for densities near the triple point value, so that the first order term alone is sufficient for quantitative accuracy.

The success of the BH and WCA theories for dense liquids of simple, spherical molecules, occurs because at these high densities the liquid structure is largely determined by the repulsive forces. Thus the radial distribution function, $g(r)$, as determined by molecular simulation for the LJ fluid and for a fluid whose molecules interact with only the repulsive part of the LJ potential are almost the same for densities near the triple point [41]. If the pair potential is written as $u(r)=u_{0}(r)+u_{p}(r)$, so that $\lambda=0$ corresponds to the reference potential and $\lambda=1$ to the full (LJ) potential, it is easy to show that the difference in the Helmholtz energy $A$ for the LJ fluid and the reference fluid is given by

$$
A \quad A_{0}=2 \quad N_{0}^{1} d \quad d r r^{2} u_{p}(r) g(r)
$$

If the difference between the radial distribution functions for the full and for the repulsive LJ fluid are negligible, $g$ can be replaced by $g_{0}$ in eqn. (7), so that the right side of this equation is the first order term. This condition is fulfilled at densities near the triple point for the LJ and similar potentials, but not at lower densities, for example near the critical point, where the first order theory is less accurate [41]. 
The Principle of Corresponding States was first proposed by van der Waals in 1881 [42] as a result of his equation of state,

$$
P_{r}=f\left(T_{r}, V_{r}\right)
$$

where $P_{r}=P / P_{c}, T_{r}=T / T_{c}$ and $V_{r}=V / V_{c}$ are the reduced pressure, temperature and volume, made dimensionless with the critical constants, and $f$ is a universal function. It is straightforward to show that eqn. (8) follows for any two-parameter equation of state, thus freeing the principle from the assumption of van der Waals equation. Equation (8) predicts that when plotted in these dimensionless units the pressure-temperature diagram for a pure substance is a universal diagram. This relationship is followed with great accuracy for the heavier inert gas liquids, $\mathrm{Ar}, \mathrm{Kr}$ and $\mathrm{Xe}$, and to a good approximation by other liquids of small, nonpolar molecules such as $\mathrm{N}_{2}$ and $\mathrm{CH}_{4}$. Equation (8) makes it possible to predict the vapor pressure curve, including the critical and normal boiling points, of liquids for which no measurements exist, based on experimental data for other liquids. The principle found almost immediate use in this way by Zygmund Wreblewski [43], who compared $(p, v, T)$ isotherms of hydrogen, oxygen and nitrogen and thus predicted that the critical point of hydrogen must be below $40 \mathrm{~K}$ (much later measurements found the critical temperature to be $33.2 \mathrm{~K}$ ), ten years before it was liquefied by Sir James Dewar, working at the Royal Institution in London [44]. Dewar was greatly impressed by the practical usefulness of the corresponding states principle in guiding experiments on cryogenic gases, going so far in his address to the British Association [45] to call it "the most powerful physical principle in the field to be discovered since Carnot's theorem" [46].

That the Principle of Corresponding States arises from similarity in the behavior of the molecules themselves was quickly realized by Heike Kammerlingh Onnes, then an assistant with Johannes Bosscha at the Delft Polytechnic, who had heard of van der Waals's principle prior to his 1881 publication [42]. He proposed that the conditions for the principle to be obeyed were $[47,48,49]$ :

First, the molecules of the different substances are completely hard elastic bodies of common shape; secondly that the long-range forces that they exert emanate from corresponding points and are proportional to the same function of the corresponding 
separations of these: and thirdly, that the absolute temperature is proportional to the mean kinetic energy of the translational motion of the molecules.

Given that in 1880 the nature and origin of intermolecular forces was not understood, and had to await the much later work of London and others, Kammerlingh Onnes showed remarkable prescience in his analysis of the underlying basis of the principle. It was nearly 60 years later before a derivation of the corresponding states principle based on statistical mechanics became available.

Derivation of the Principle from statistical mechanics was carried out in the late 1930's, first for gases by Jan de Boer and Antonius Michels in 1938 [50] working at the University of Amsterdam, and in the following year by Kenneth Pitzer [51] at the University of California at Berkeley for the more general case of liquids and dense fluids. The derivation relies on two assumptions: (a) the translational motion of the molecules is classical (Kammerlingh Onnes third condition), and (b) the total intermolecular potential energy, $\mathcal{U}=\varepsilon \Phi\left(\frac{x_{1}}{\sigma}, \frac{y_{1}}{\sigma}, \frac{z_{1}}{\sigma}, \frac{x_{2}}{\sigma}, \frac{y_{2}}{\sigma}, \frac{z_{2}}{\sigma}, \ldots\right)$, can be described by two parameters, an energy, $\varepsilon$ and a length, $\sigma$ that is characteristic of the fluid considered. Here $\Phi$ is a universal function. This is essentially Kammerlingh Onnes second condition, expressed in more explicit form. With these assumptions the equation of state can be written as

$$
P^{*}=f\left(T^{*}, V^{*}\right)
$$

where $P^{*}=P^{3} /, \quad T^{*}=k T /$ and $V^{*}=V / N_{A}{ }^{3}$ with $N_{A}$ the Avogadro number and $f$ is a universal function. It follows from eqn. (9) that if the pressure-temperature diagram is plotted as $P^{*}$ vs. $T^{*}$ there is a universal vapor pressure line, and the conditions at the critical point are $T_{c}{ }^{*}=c_{1}, V_{c}{ }^{*}=c_{2}$ and $P_{c}{ }^{*}=c_{3}$ and where $c_{1}, c_{2}$ and $c_{3}$ are universal constants whose values for the heavier inert gases are $c_{1} \sim 1.29, c_{2} \sim 2.82$ and $c_{3} \sim 0.133$, based on potential parameters determined from gas second virial coefficient data [52]. Thus the potential parameters are easily related to the critical constants by $/ k=T_{c} / c_{1}, \quad{ }^{3}=V_{c} / c_{2} N_{A}$ and $\quad I^{3}=P_{c} / c_{3}$, and the molecular principle of corresponding states of eqn. (9) is entirely equivalent to the macroscopic form of eqn. (8).

\section{Polar Liquids}


The first serious attempt at a theory of polar liquids was made in 1951 by John A. Barker, working at the Commonwealth Scientific and Industrial Research Organization in Melbourne, in a Letter to the Editor of the Journal of Chemical Physics [53]. This work, Barker's first paper, was inspired by Longuet-Higgins' landmark paper on conformal solution theory for mixtures of spherical molecules, whose pair intermolecular potentials were of the form $u(r)=f(r /)$. He considered a mixture of two components, one nonpolar and composed of spherical molecules, the other having a point dipole of moment $\mu$, so that the total potential energy was

$$
\mathcal{U}=\mathcal{U}_{0}+\mathcal{U}_{p}=\sum_{\alpha} \sum_{\beta} \varepsilon_{\alpha \beta} f\left(\frac{r_{\alpha \beta}}{\sigma_{\alpha \beta}}\right)+\sum_{\kappa} \sum_{\lambda} \frac{\mu^{2} \phi\left(\omega_{\kappa}, \omega_{\lambda}\right)}{r_{\kappa \lambda}}
$$

where the last term on the right represents the sum of dipole-dipole interactions for the dipolar molecules and $(, \quad)$ is the usual dipole-dipole function of molecular orientations, $\omega_{\kappa}$ and $\omega_{\lambda}$. Barker treated the dipole-dipole term in eqn. (10) as a perturbation and expanded the configuration integral and hence the Helmholtz energy, $A$, in powers of this, to obtain

$$
A=A_{0}+A_{1}+A_{2}+\ldots=A_{0}+\left\langle\mathcal{U}_{p}\right\rangle_{0}-\frac{1}{2} \beta\left\langle\mathcal{U}_{p}^{2}\right\rangle_{0}+\ldots
$$

where $=1 / k T$ and $\langle\ldots\rangle_{0}$ signifies an ensemble average over the reference system of spherical molecules. The reference term $A_{0}$ is the Helmholtz energy of a liquid of spherical molecules interacting with a pair potential $u_{0}(r)=\langle u(12)\rangle_{12}$, i.e. an unweighted average over the orientations of the full potential. The first order term $\left\langle\mathcal{U}_{p}\right\rangle_{0}$ vanishes for the dipole-dipole interaction, so that the second order term is the first non-vanishing one. Barker showed that the second order term could be related to thermodynamic properties of a pure fluid of molecules with the reference fluid parameters, $\varepsilon_{x}$ and $\sigma_{x}$, but did not carry out any calculations. In a 1953 paper [54] Barker extended this treatment to the case of polar fluids in which the molecules possessed polarizability in addition to the permanent dipole, and showed through numerical calculations that the polarizability had a significant effect on the thermodynamic properties. 


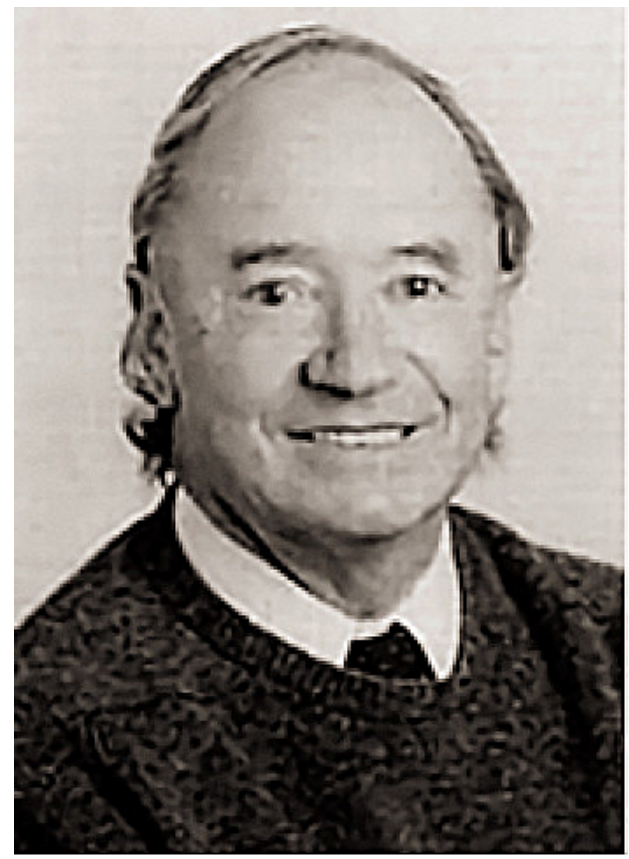

Figure 1. John Adair Barker.

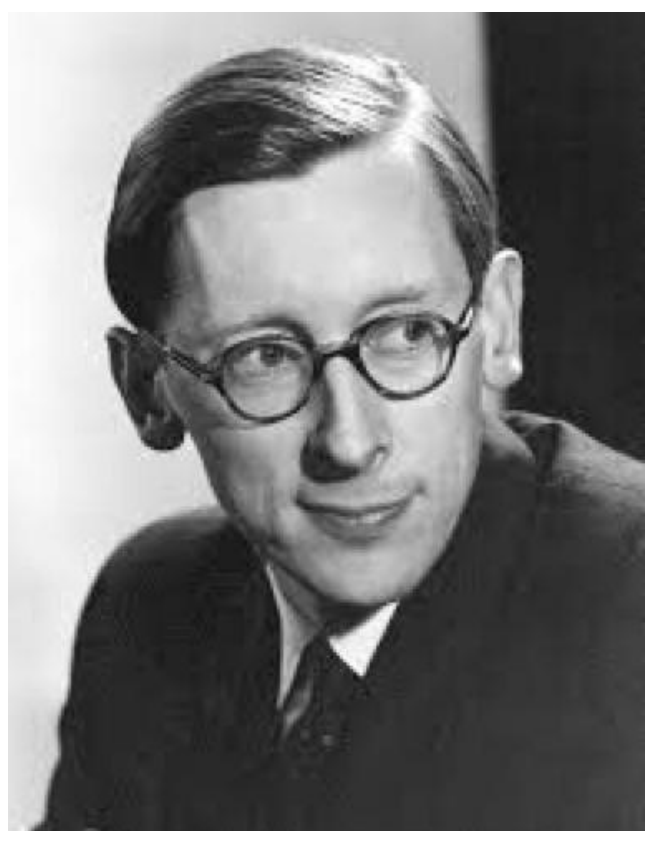

Figure 2. John A. Pople.

John Pople, while a research fellow at Trinity College, Cambridge also obtained the second order perturbation expansion of eqn. (11) for a fluid of dipoles in 1952 [55], using a lattice model approach. Pople appears to have been unaware of Barker's earlier work at this time. The following year, Pople presented a more rigorous off-lattice perturbation theory [56] at the Faraday Discussion on The Equilibrium Properties of Solutions of Non-Electrolytes, held at the Royal Institution in London. In this paper he gave for the first time the expression for the second order term in the free energy expansion as an integral over the pair correlation function of the reference system for a perturbing potential that was a sum of electrostatic terms. In this paper he also reported some calculations of the influence of dipolar interactions on thermodynamic properties for several mixtures. However, in 1953 there was no satisfactory way to calculate the pair correlation function, $g_{0}(r)$, for the reference fluid of spherical molecules. Pople resorted to lattice theory to overcome this difficulty, but the resulting agreement with experiment was poor. In a seminal paper published in 1954 Pople [57] presented a more general version of the theory, in which he assumed that the molecules were axially symmetric and that the intermolecular pair potential could be expressed as a series of spherical harmonic terms, and 
obtained a general expression for the second order contribution to free energy in terms of pair, $g_{0}(r)$, and triplet, $g_{0}\left(r_{12}, r_{13}, r_{23}\right)$, correlation functions for the reference system,

$$
\begin{aligned}
A_{2}= & \frac{1}{4} \quad{ }^{2} d \mathbf{r}_{1} d \mathbf{r}_{2} g_{0}(12)\left\langle u_{p}(12)^{2}\right\rangle_{12} \\
& \frac{1}{2} \quad{ }^{3} d \mathbf{r}_{1} d \mathbf{r}_{2} d \mathbf{r}_{3} g_{0}(123)\left\langle u_{p}(12) u_{p}(13)\right\rangle_{123}
\end{aligned}
$$

where $\langle\ldots\rangle_{12 \ldots}$ signifies an unweighted average over the molecular orientations shown. Here $u_{p}$ is a sum of spherical harmonic terms $u\left(\ell_{1} \ell_{2} \ell\right)$, while the reference fluid is one whose molecules interact with $u(000)$. Pople gave explicit expressions for anisotropic induction, dispersion and overlap potentials, as well as electrostatic ones. When $u\left(\ell_{1} \ell_{2} \ell\right)$ is 'multipole-like', that is $\ell_{1}, \ell_{2}$ and $\ell$ are all non-zero, the last three-body term on the right of eqn. (12) vanishes, simplifying the second order term considerably. In a subsequent paper [58] Pople applied this theory to the calculation of second virial coefficients.

Thus a formal, off-lattice theory for liquids of non-spherical molecules was developed in the period 1951-54, but application of this was limited by the lack of a successful theory of simple liquids, and consequent difficulty in calculating the properties of the reference system, $A_{0}$ and $g_{0}$. At that time the only candidates were the lattice theories and the corresponding states theory. Lattice theories of liquids based on statistical mechanical models appeared in the 1930's [59,60] and were based on the premise that the structure of liquids was more similar to that of crystalline solids, rather than that of dense gases (as had been implied in the approaches of van der Waals, Hildebrand and others), based on X-ray date available at the time. Lattice theories quickly fell out of favor by physicists, but persisted much longer among chemists and engineers, and were pursued and popularized by several groups in the period 1937 to 1964, notably Eyring and coworkers at the University of Utah, Fowler and Guggenheim at Cambridge University and the University of Reading, and Progogine and coworkers at the Free University of Brussels. Thus Fowler and Guggenheim in their monograph Statistical Thermodynamics published in 1939, declare [61]: 
We are therefore driven to the conclusion that a liquid is much more like a crystal than like a gas, and the structure that we shall accept as the most plausible for a liquid is conveniently referred to as quasi-crystalline.

By placing the molecules on lattice points, or tethered to lattice points in some way, the difficult integrals that must be evaluated in the configurational partition function are replaced by simple sums that are readily calculated. No molecular simulation studies of simple liquids were available to test these theories until the 1960 's, so that the equations were tested against experimental data available at the time. In the Preface to his monograph Mixtures E.A. Guggenheim wrote in 1951 [62]:

Where comparison is possible (with experiment) the result is nearly always surprisingly gratifying.

However, such comparisons involved the fitting of both lattice and potential parameters to experiment, and it was later shown that the apparent agreement was largely illusory. The first unambiguous test of the lattice theory was reported by E. Brian Smith and Kenneth R. Lea [63,64], working at the Physical Chemistry and Clarendon Physics Laboratories at Oxford University. They carried out Monte Carlo simulations of a binary hard sphere mixture in which the ratio of diameters was ${ }_{B B} /{ }_{A A}=5 / 3$. The lattice theory for hard spheres takes a particularly simple form [64]. Comparison with the MC results showed that the lattice theory gave quite the wrong density dependence of the compressibility factor. Since the Smith and Lea papers were focused on testing the virial series equation of state, this important test of the lattice theory was largely overlooked at the time, but later simulation results confirmed its failure for liquids [refs. 3,4,8 and references therein]. A major flaw in such theories is the underestimation of the entropy, as the lattice has too much order. A further and even more serious defect is that placing molecules on lattice sites makes them distinguishable by virtue of their position in space, in violation of quantum mechanics, which teaches us that molecules of the same species in a fluid are indistinguishable. 
In the 1950's corresponding states theory provided a more useful route for the calculation of thermodynamic properties of liquids having non-central intermolecular forces. Thus, in 1953 Cook and Rowlinson [65] showed that if the non-central part of the interaction is multipole-like and not too large, it is possible to equate the thermodynamic functions to those for a liquid of spherical molecules that interact with an effective potential energy, $u^{e f f}(r)$ that is temperature-dependent. This can be seen by considering the series $A=A_{0}+A_{2}+\ldots$ of eqn. (11), terminated at the second-order term. For a multipole-like potential, $u_{p}\left(\mathbf{r}, 1_{1}{ }_{2}\right)$, eqn. (11) becomes

$$
\begin{aligned}
& A=A_{0}+A_{2}+\ldots=A_{0} \quad \frac{1}{4} \quad{ }^{2} d \mathbf{r}_{1} d \mathbf{r}_{2} g_{0}(r)\left\langle u_{p}(12)^{2}\right\rangle_{12}+\ldots \\
& =A_{0} \quad \frac{1}{2}{ }^{2} d \mathbf{r}_{1} d \mathbf{r}_{2} g_{0}(r) u_{1}^{e f f}(r)+\ldots
\end{aligned}
$$

where

$$
u_{1}^{e f f}(r)=\frac{1}{2 k T}\left\langle u_{p}(12)^{2}\right\rangle_{12}
$$

This result for a liquid of non-spherical molecules can be compared with a liquid of spherical molecules interacting with a pair potential $u^{\text {eff }}(r)=u_{0}(r)+u_{1}^{\text {eff }}(r)$, where $u_{0}$ is the reference potential of eqn. (11), i.e. an unweighted average over the orientations of the full potential for the non-spherical molecules, and $u_{1}^{\text {eff }}$ is given by eqn. (14). The Helmholtz energy of this liquid of spherical molecules is given by

$$
A=A_{0}+A_{1}^{e f f}+\ldots=A_{0} \quad \frac{1}{2}{ }^{2} d \mathbf{r}_{1} d \mathbf{r}_{2} g_{0}(r) u_{1}^{e f f}(r)+\ldots
$$

Thus, to first non-vanishing order eqns. (13) and (15) give the same result, and the thermodynamic properties of the liquid of non-spherical molecules are the same as those for the liquid of spherical molecules with pair potential $u^{\text {eff }}(r)=u_{0}(r)+u_{1}^{e f f}(r)$ provided that the non-central part of the potential is sufficiently weak. If the reference fluid with potential $u_{0}$ is taken to be the Lennard-Jones model, this approach is especially simple if the effective central potential of eqn. (14) has the same $r$-dependence as one of the terms in the LJ potential. It is then possible to rearrange the effective central potential into the LJ form. This is the case for the dipole-dipole interaction and for the dipole-induced dipole 
interaction, for both of which $\left\langle u_{p}(12)^{2}\right\rangle, \mu r^{6}$. In this case the effective central potential is;

$$
u^{e f f}(r)=4\left[\left(\frac{-}{r}\right)^{12}\left(\frac{-}{r}\right)^{6}\right] \frac{4}{3 k T r^{6}} \frac{2^{2}}{r^{6}}=4^{e f f}\left[\left(\frac{e f f}{r}\right)^{12}\left(\frac{e f f}{r}\right)^{6}\right]
$$

where $\mu$ and $\alpha$ are the dipole moment and polarizability, and

$$
\begin{aligned}
& e f f=F^{2} \\
& e f f=F^{1 / 6}
\end{aligned}
$$

where

$$
F(T)=\left(1+\frac{4}{12 k T^{6}}+\frac{2}{2^{6}}\right)
$$

It then follows that the polar fluid will obey the simple corresponding states relationship of eqn. (9),

$$
P *=f_{L J}(T *, V *)
$$

where $f_{L J}$ is the spherical Lennard-Jones equation of state, and the effective potential parameters are used in the definition of reduced pressure, temperature and volume, i.e. $T=k T /{ }^{\text {eff }}, P=P^{\text {eff } 3} /{ }^{\text {eff }}, V=V / N_{A}{ }^{\text {eff } 3}$. Since $f_{L J}$ is the same function as that of eqn. (9) for spherical molecules, by the argument given below that equation we can replace ${ }^{e f f}$ and eff by critical constants, using $\underset{c}{e f f} / k=T_{c} / c_{1}, \quad \underset{c}{e f f 3}=V_{c} / c_{2} N_{A}$ and $\underset{c}{e f f} / \underset{c}{e f f 3}=P_{c} / c_{3}$ where $c_{1}, c_{2}$ and $c_{3}$ are the same constant values as for simple spherical LJ molecules. Here $\underset{c}{e f f}$ and $\underset{c}{e f f}$ are the effective potential parameter values at the critical temperature, i.e. ${ }_{c}^{e f f}=F_{c}^{2}$ and ${ }_{c}^{e f f}=F_{c}{ }^{1 / 6}$. Thus the macroscopic form of the equation of state is

$$
P_{r}=f_{L J}\left(T_{r}, V_{r}\right)
$$

where

$$
T_{r}^{\prime}=\frac{T}{T_{c}}\left(\frac{F_{c}}{F}\right)^{2} \quad V_{r}^{\prime}=\frac{V}{V_{c}}\left(\frac{F_{c}}{F}\right)^{1 / 2} \quad P_{r}^{\prime}=\frac{P}{P_{c}}\left(\frac{F_{c}}{F}\right)^{5 / 2}
$$

Here $F$ is evaluated at temperature $T$ and $F_{c}$ is evaluated at temperature $T_{c}$. According to eqn. (20) the polar fluid should obey the same equation of state, $f_{L J}$, as the simple fluids, provided that the reduced temperature, etc., are defined according to eqn. (21). Eqn. (20)- 
(21) should be obeyed provided that the dipole moment is not too large, or the temperature too low. One test of this is that the reduced critical temperature, critical volume and critical pressure should each equal the same constants, $c_{1}, c_{2}$ and $c_{3}$, as for the simple inert gas liquids. For the moderately polar liquids $\mathrm{HCl}, \mathrm{SO}_{2}, \mathrm{CH}_{3} \mathrm{Cl},\left(\mathrm{CH}_{3}\right)_{2} \mathrm{CO}$, and $\mathrm{C}_{2} \mathrm{H}_{5} \mathrm{OH}$, with dipole moments ranging from $1.04 \mathrm{D}\left(\mathrm{CHCl}_{3}\right)$ to $1.90 \mathrm{D}\left(\mathrm{CH}_{3} \mathrm{Cl}\right)$, the average value of these 'constants' are [52] $c_{1}=1.30, c_{2}=3.07$ and $c_{3}=0.116$, close to the values for the inert gas liquids of $c_{1}=1.29, c_{2}=2.82$ and $c_{3}=0.133$. A further test is provided by the reduced vapor pressure, $P_{r}^{\text {sat }}$, which should fall on the same curve as the simple fluids (inert gas liquids) when plotted against inverse reduced temperature, $T_{r}$. A test of this for methyl chloride ( $\mu=1.90 \mathrm{D}$, a fairly large dipole moment) is shown in Figure 3. Very good agreement with the heavier inert gas liquids is found for the higher temperatures, but deviations start to be observed for temperatures below $\sim T_{r} \sim 0.6$. A similarly simple effective LJ model can be used $[65,66,67]$ for molecules with anisotropic London dispersion interactions $\left(\left\langle u_{p}(12)^{2}\right\rangle_{12} \mu r^{12}\right)$.

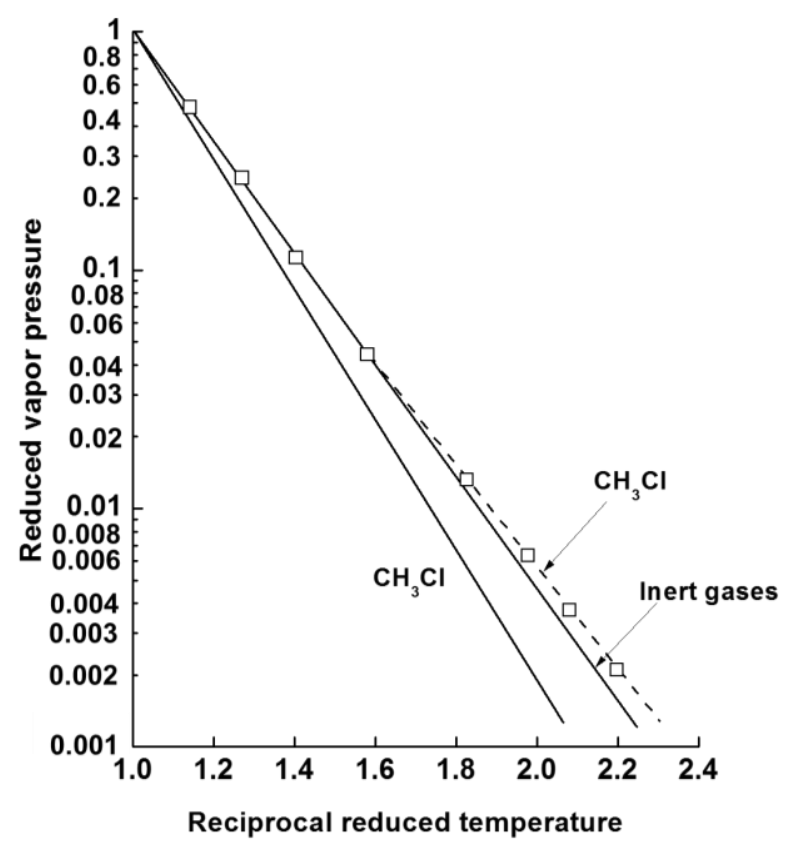

Figure 3. Reduced vapor pressures for methyl chloride as predicted by eqns. (20) \& (21) (points and dashed line), compared with the simple corresponding states theory of eqn. (8) for the inert gases (upper solid line) and methyl chloride (lower solid line). After ref. 52. 


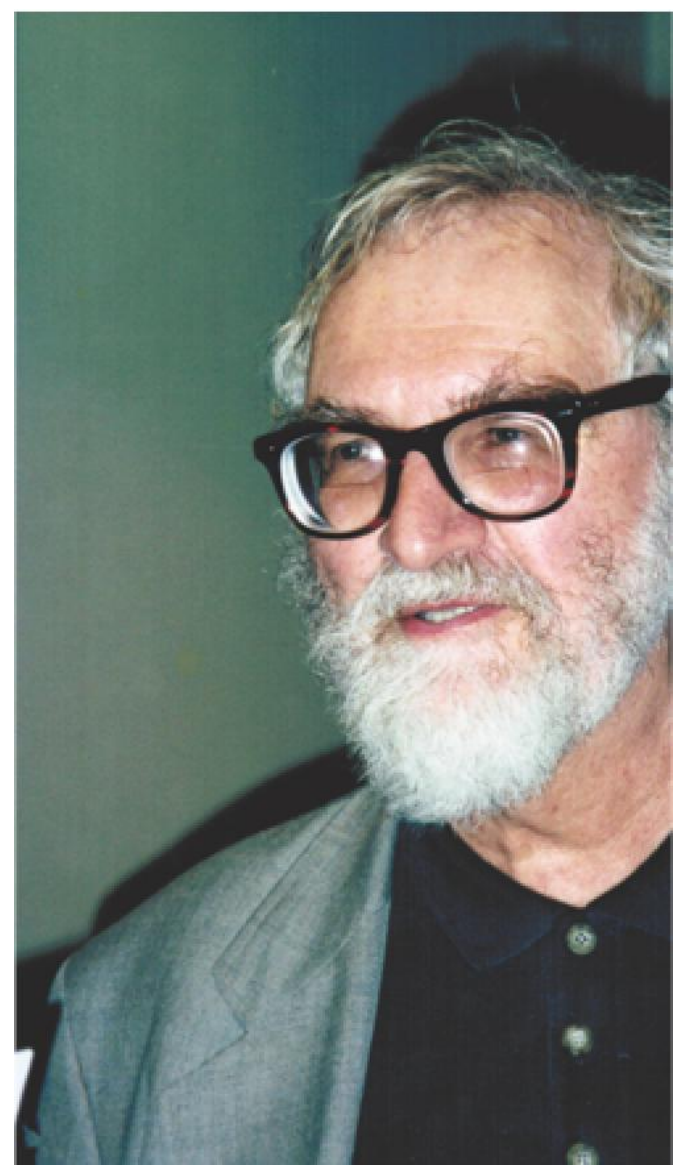

Figure 4. George Stell, ca. 2000 [Photo courtesy of Carol K. Hall]

Although the groundwork for a perturbation theory for polar liquids had been laid by Barker, Pople, Rowlinson and others in the early 1950's, further developments had to await the development of a successful theory for simple liquids of spherical molecules. Such theories did not appear until the period 1967-1971. Thus it was more than twenty years after Barker's 1951 paper proposing a perturbation expansion for polar liquids that this subject was taken up anew, by George Stell (Figure 4) and coworkers at the State University of New York at Stony Brook, and independently by Keith Gubbins and Chris Gray, at Cornell University and the University of Guelph, respectively. In 1972 Gubbins and Gray [68] presented the perturbation expansion for the pair correlation function, $g(\mathbf{r}, \quad 1,2)=g_{0}(r)+g_{1}\left(\mathbf{r}, 1_{1}, 2\right)+g_{2}\left(\mathbf{r}, 1_{1}, 2\right)+\ldots$, and evaluated the terms up to $g_{2}$. This series corresponds to the Barker expansion of eqn. (11) for the Helmholtz energy up to the 
third order term, $A_{3}$, as seen from the molecular analog of eqn. (7). They reported calculations of the pair correlation function for a quadrupolar liquid with pair potential consisting of a LJ term and a quadrupole-quadrupole term (Figure 5), and showed that even for a relatively small quadrupole moment, $Q=Q /\left({ }^{5}\right)^{1 / 2}=0.28$, the quadrupole term had a large effect on $g(12)$. At almost the same time, Stell et al. [69] examined the Barker expansion of the Helmholtz energy, eqn. (11), up to the third order term, $A_{3}$, choosing as the reference a LJ fluid, and reported calculations for a Stockmayer fluid (LJ plus point dipole term) with a dipole moment of strength $=I\left(3^{3}\right)^{1 / 2}=1.0$. They showed that dipoles of this magnitude have a significant effect on the thermodynamic properties over the whole liquid region. In the following few years a number of authors tested this perturbation theory against molecular simulations for both the pair correlation function [70] and the thermodynamic functions [71-75]. An example of such a test is given in Figure 6, which shows a comparison of the Barker expansion of eqn. (11) up to the $A_{3}$ term with Monte Carlo simulations for a pair potential consisting of the sum of a $L J$ term and either the point dipole-dipole or quadrupole-quadrupole potential. The series to the third order term is seen to work well for small and moderate values of the reduced multipole moment, but starts to deviate from the simulations for values greater than about 0.7 or 0.8 .

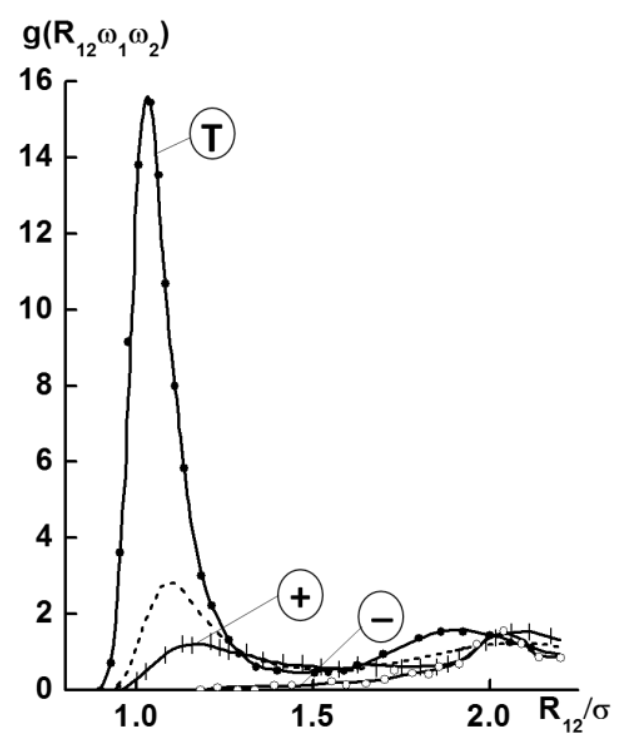


Figure 5. The angular pair correlation function, $g\left(\mathbf{r}_{12}, 1_{2},{ }_{2}\right)$, for a liquid of molecules interacting with a LJ plus point quadrupole-quadrupole term for $Q=Q /\left({ }^{5}\right)^{1 / 2}=1.0$ at $={ }^{3}=0.80, T=0.719$. The dashed curve is $g_{0}(r)$ for the LJ fluid, and points and solid lines are $\mathrm{MC}$ results for the T, cross and end-to-end orientations. Adapted from ref. 70.

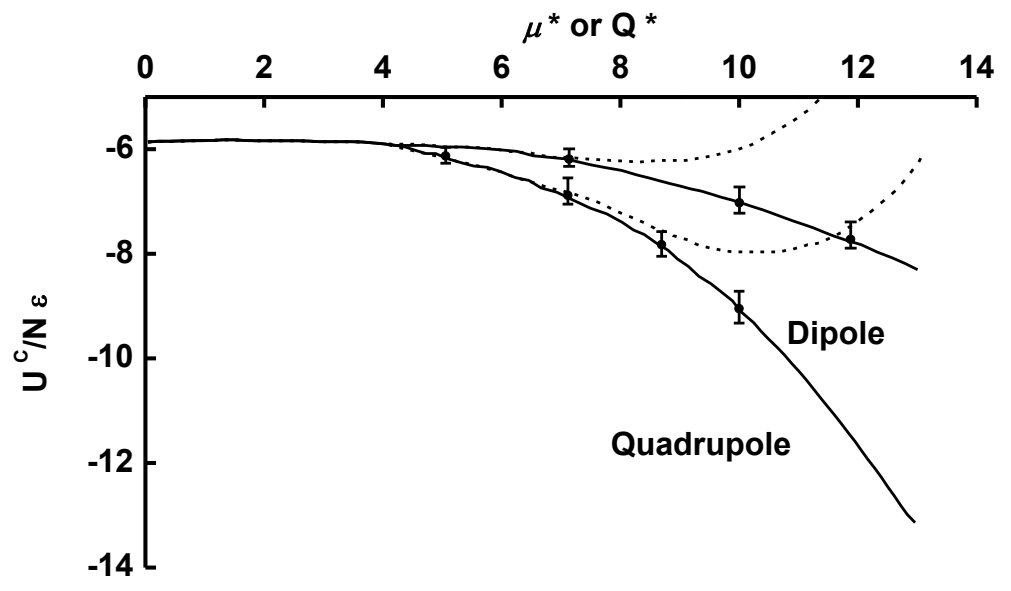

Figure 6. The configurational part of the internal energy for a liquid in which the molecules interact with a pair potential $u_{L J}+u_{p}$, where $u_{p}$ is either the point dipole-dipole or quadrupole-quadrupole potential. Dashed lines are the Barker expansion of eqn. (11), solid lines are the Padé approximant of eqn. (22), and points are MC simulation values [70]. Here $\mu^{*}=\mu /\left(\varepsilon \sigma^{3}\right)^{1 / 2}$ and $Q^{*}=Q /\left(\varepsilon \sigma^{5}\right)^{1 / 2}$. After ref. 75 .

That the Barker expansion of eqn. (11) fails to converge for large values of the multipole moments is readily understood from Figure 5. Eqn. (7) implies that rapid convergence of the perturbation expansion requires a reference system having a structure, in the form of $g_{0}(12)$, that is similar to the angular pair correlation function for the real system, $g(12)$. As shown in Figure 5, this is far from being the case when electrostatic forces are present, the most probable $\mathrm{T}$ orientation having a first peak that is more then 5 times that of the reference system. Moreover, it is clear that this will be the case for any reference system of spherical molecules. 
Although the series of eqn. (11) converges poorly for strong electrostatic forces, for multipolar potentials the $A_{2}$ and $A_{3}$ terms are of opposite sign, and so it is possible to approximately re-sum the series using a 0/1 Padé approximant,

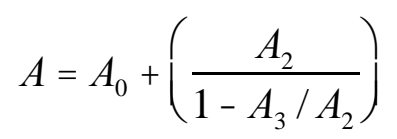

Expansion of the second term on the right of this expression recovers the series of eqn. (11) up to $A_{3}$, and so gives the weak perturbation limit, $A=A_{0}+O\left(u_{p}^{2}\right)$, correctly. In the large perturbation limit (e.g. very strong dipoles or quadrupoles) the Padé predicts a linear dependence on the perturbing potential, $A=A_{0}+O\left(u_{p}\right)$. Such dependence for strong perturbations is reasonable. Thus, if the perturbing potential is due to a dipolar interaction, for very strong dipole moments the relative molecular orientations will become fixed, and further increase in dipole strength will cause the Helmholtz energy to scale as $u_{p}$. Not only does eqn. (22) display the correct scaling behavior for large perturbations, it predicts the $O\left(u_{p}\right)$ term with surprising accuracy. Sullivan et al. [76] compared the Padé of eqn. (22) with the infinite series of eqn. (11) for a rigid polar lattice, for which the terms $A_{n}$ can be calculated to high order, and found that as $/\left(3^{3}\right)^{1 / 2} \rightarrow \infty$ eqn. (22) agreed within $0.15 \%$ with the exact result. Equation (22) turns out to be accurate for strong multipole moments, as shown in tests against molecular simulations for dipolar and quadrupolar liquids [71,72,74-77], and as seen in Figure 6. Eqn. (22) was first published in the Master's thesis of H. Narang in 1972 [78], but was little noticed until it appeared in later publications by Rushbrooke et al. [79] and in particular the 1974 paper of Stell, Rasaiah and Narang [80].

The good agreement between the Padé and molecular simulation results meant that the theory could be tested against experimental results with greater confidence, such comparisons being primarily a test of the intermolecular potential models used. However, there was a dearth of suitable experimental studies with which to make such tests in the early 1970's. It was largely thanks to Lionel Staveley, working at the Inorganic Chemistry Laboratory at Oxford University, and somewhat later Jorge Calado (formerly a student of Staveley's) at the Instituto Superior Técnico in Lisbon, that this situation was rectified by a 
series of careful studies involving liquids of small, non-spherical molecules carried out and reported in the period from 1976 to the mid-1980's. These liquids were chosen to have a range of shapes, multipole moments and polarizabilities, and included diatomics $\left(\mathrm{N}_{2}, \mathrm{CO}\right.$, $\mathrm{HCl}, \mathrm{HBr}$, etc.), triatomics $\left(\mathrm{CO}_{2}, \mathrm{~N}_{2} \mathrm{O}\right)$, and small polyatomics $\left(\mathrm{CH}_{4}, \mathrm{CF}_{4}, \mathrm{CH}_{3} \mathrm{Cl}_{1} \mathrm{C}_{2} \mathrm{H}_{4}\right.$, etc.). Comparisons of these data with the Padé of eqn. (22) followed in the period from the late 1970 's until late 1980's, using intermolecular potential functions that included the lower multipole and induction terms. Generally good agreement was found provided that the molecules were not too non-spherical in shape. In the case of liquid mixtures, when the Lorentz-Berthelot combining rules were used for the LJ parameters, agreement with experiment was much improved over the use of spherically symmetric potentials $[81,82]$. However, quantitative agreement usually required the fitting of one unlike pair parameter, ${ }_{A B}$. These tests against experiment have been reviewed elsewhere [8,83].

By the late 1970's the important influence of the permanent electric moments on the thermodynamics and structure of liquids was well established, but it was not until the end of that decade that the importance of induction forces (the polarization of molecules due to the electric field of nearby molecules with permanent moments) was fully realized. Early perturbation theory studies of polarizable systems, for example those of McDonald [73] and Larsen et al. [84], failed to describe the effect of anisotropy of the polarizability, or to take into account many-body induction forces (four or more molecules), which are very difficult to include in conventional perturbation treatments since they require knowledge of 4-body and higher correlation functions for the reference system. Subsequent molecular simulation [85] and theoretical $[86,87]$ studies showed that these higher order many-body induction interactions make important contributions to both the thermodynamic and structural properties of polar liquids.

The difficulties in accounting for induction effects using conventional perturbation theory were overcome by Michael Wertheim [86], who applied resummation (or renormalization) methods to the expansion to achieve a tractable and accurate theory that accounted for both the higher many-body induction interactions and the anisotropy of the polarizability, . This theory is usually referred to as the renormalized perturbation theory (RPT). The central idea of Wertheim's RPT is that most of the molecules in the liquid 
will have a total dipole moment (permanent plus induced) that is close to the average total dipole moment, , rather than to the permanent (gas-phase) dipole moment . In typical cases of polar liquids the magnitude of the total moment, , can exceed that of the permanent moment by $20-50 \%$, so that the resulting induction effects are large. The perturbation expansion is then carried out in terms of and , rather than and . This corresponds to what Wertheim refers to as first level of renormalization (1-RPT). He also considers a second level renormalization (2-RPT), in which the polarizability, , is renormalized in addition to the dipole moment. In most cases, however, the 2-RPT theory gives results that are only a little different from those of 1-RPT.

The RPT is found to give good agreement with Monte Carlo simulation results that account for both many-body induction interactions and anisotropy of the polarizability $[85,88]$. The theory has been extended to liquid mixtures of polar-polarizable molecules by Venkat Venkatasubramanian and coworkers [89], who reported calculations showing the large effect of induction forces on both excess thermodynamic properties and vapor-liquid equilibria for typical values of dipole moments and polarizabilities. The theory has also been extended to polarizable dipolar-quadrupolar molecules [90] and to polarizable quadrupolar molecules [91], and has been compared to experimental binary mixture results for excess thermodynamic properties and vapor-liquid equilibria $[88,89,90,92,93]$ and for liquid-liquid equilibria $[88,93]$. In addition to these thermodynamic properties, it is possible to calculate the high-frequency dielectric constant, , from the 2-RPT version of the theory [91].

The derivation of RPT by Wertheim relies on knowledge of graph analysis and is rather complicated. A simpler non-graphical derivation of RPT has been presented by Joslin et al. [91].

\section{Associating Liquids}

The perturbation treatments considered in the last two sections are appropriate for molecules that interact with dispersion, overlap and weak to moderate electrostatic and induction forces. Such molecules have pair interactions with energies up to 2 or $3 \mathrm{~kJ} / \mathrm{mol}$ in the liquid phase. However, many liquids exhibit molecular association due to hydrogen-bonding, charge 
transfer, or other forms of complexing, and have interaction energies in the range $15-40 \mathrm{~kJ} / \mathrm{mol}$, an order of magnitude larger than for the simpler liquids. In 1908 Dolezalek [94] proposed a 'chemical' theory of mixtures, in which the components were assumed to react chemically, so that any associated complexes were treated as new chemical species, the components forming an ideal mixture. In such a treatment acetic acid would be considered to be a mixture of $\mathrm{CH}_{3} \mathrm{COOH}$ monomers and $\left(\mathrm{CH}_{3} \mathrm{COOH}\right)_{2}$ dimers, with concentrations, $c$, related by $\mathrm{K}=c_{\left(\mathrm{CH}_{3} \mathrm{COOH}\right)_{2}} / c_{\mathrm{CH}_{3} \mathrm{COOH}}^{2}$ where $K$ is the equilibrium constant. The ideal mixture assumption was omitted in later versions of this approach, so that concentrations were replaced by activities [95]. Dolezalek's theory was widely criticized by van Laar [96,97], Hildebrand [98], Scatchard [99] and others. While the chemical approach is not unreasonable for acetic acid dimerization, for which evidence of the dimers is clearly seen in spectra, Dolezalek did not know where to stop, and applied his idea to mixtures where no association occurs. As Hildebrand remarked [98]:

The advocates of the Dolezalek theory are often led to assume association in liquids which, according to all other criteria, are entirely normal or unassociated. The most striking example....is found in a paper by Dolezalek (Z. Physik. Chem., 93, 585 (1918)) in which liquid solutions of nitrogen and argon are "explained" by assuming polymerization of the argon to form double molecules!

The chemical theory was criticized on other grounds, including the incorrect assumption that the intermolecular interactions between unlike molecules is the arithmetic mean of the like-pair interactions. The chemical approach is often of limited usefulness in practice, because the 'reactions' and the equilibrium constants are often unknown.

In physical theories association occurs as a result of intermolecular forces and can be treated by the methods of statistical mechanics. Such an approach has the advantages that the theory can be tested against molecular simulations for the same well-defined potential models, and it is not necessary to postulate any 'chemical reactions' in advance. The earliest such theories were lattice models [100-103]. By postulating weak bonds between unlike molecular pairs they were able to qualitatively describe mixtures that exhibit a closed solubility loop on the $T-x$ phase diagram, with both an upper and a lower critical point. However, such theories underestimate the entropy and cannot provide an accurate description of the thermodynamics. Among the first off-lattice treatments of associating fluids were those of Andersen [104,105], who proposed a cluster expansion in terms of the number density for molecules having a highly 
directional attraction site embedded in a repulsive core. Andersen's work prompted later theories [106-109] that made use of fugacity expansions, which lead to faster convergence. In 1980 Høye and Olaussen [107] introduced an expansion in terms of both the total density and the monomer density, a forerunner of the multi-density formalism introduced a few years later by Wertheim that proved so successful.

The most successful and useful of the off-lattice theories was developed by Michael Wertheim (Fig. 7), working at Rutgers University, and presented in a series of papers $[110,111]$ published in the Journal of Statistical Physics in the period 1984 - 86. Wertheim considers a fluid of hard spheres each with a single off center site of short ranged attraction, so that at equilibrium the fluid contains monomers having number density ${ }_{M}$ and dimers of number density ${ }_{D}$. He then expands the logarithm of the grand partition function, $\Xi$, in both the monomer density, ${ }_{M}$, and the total density of spheres, $={ }_{M}+2_{D}$, and shows that such a dual expansion is much more tractable for such associating fluids than the usual Mayer expansion in terms of $\rho$. The dual density expansion is further simplified by invoking a number of physically reasonable steric incompatibility conditions: (a) Two molecules cannot bond at a given site; (b) No site on a given molecule can bond simultaneously to two sites on another molecule; and (c) double bonding between molecules is not allowed. If the bonding sites are of sufficiently short range, e.g. off-center square well sites with narrow wells, these conditions are easily fulfilled. The steric incompatibility conditions enable many of the complex graphs to be neglected, and following topological reduction of the remaining graphs a simple result is obtained for the Helmholtz energy. The derivation of the theory by Wertheim involves graphical expansion and relies on a detailed knowledge of graph manipulation. A simpler, more heuristic, derivation involving renormalization of the perturbation series has been given by Joslin et al. [112]. 


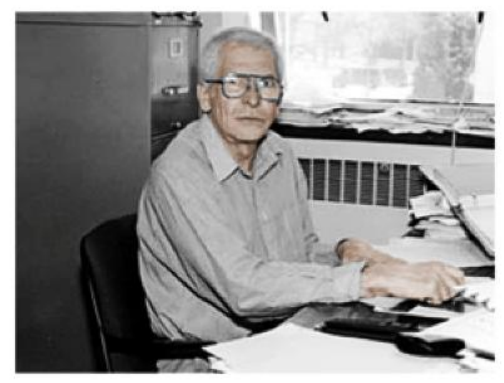

Figure 7. Michael Wertheim.

The theory was subsequently extended $[113,114]$ to molecules having a spherical core but multiple off-center bonding sites, labeled $1,2, \ldots i, \ldots n$. In this case the expansion is carried out in the densities of molecules bonded at site 1 , molecules bonded at site 2 , etc. The resulting expression for the Helmholtz energy $A$ is [114]:

$$
\frac{A \quad A_{0}}{N k T}=\sum_{i}\left(\ln X_{i} \frac{X_{i}}{2}\right)+\frac{1}{2} n
$$

where $A_{0}$ is the Helmholtz energy of the reference system of spherical molecules and $X_{i}$ is the fraction of molecules that are not bonded at site $i$, given by

$$
X_{i}=\left[1+\sum_{j} X_{j} \quad\right]^{1}
$$

where

$$
{ }_{i j}=\int\left\langle g_{0}(12) f_{i j}(12)\right\rangle_{12} d \boldsymbol{r}_{12}
$$

In eqn. (25) $g_{0}(12)$ is the reference system pair correlation function and $f_{i j}(12)=e^{u_{i j}(12) / k T} 1$ is the Mayer $f$-function for the bonding potential between sites $i$ and $j$ on two different molecules. Eqn. (23) has been found to be in excellent agreement with molecular simulations [112,115-118] for a variety of molecular models. These models usually take hard spheres or spherical LJ molecules as the reference fluid, and treat the bonding as arising from off-center square well or point charge sites. An example of such a test for the monomer mole fraction for a model consisting of hard spheres plus either one or two square well sites is shown in Figure 8. The bonding between site A in molecule 1 and site $B$ in molecule 2 is 


$$
\begin{aligned}
u_{A B}\left(r_{A B}\right) & ={ }_{A B} & & r_{A B} r_{c} \\
& =0 & & r_{A B}>r_{c}
\end{aligned}
$$

Similarly excellent agreement was found for the configurational energy and the pressure [117]. The expression for the latter is readily found by applying the thermodynamic relation, $P=(A / V)_{T}$ to eqn. (23):

$$
\frac{P}{k T}=\left(\frac{P}{k T}\right)_{0}+\left[\sum_{i}\left(\frac{\partial X_{i}}{\partial}\right)_{T}\left(\frac{1}{X_{i}} \frac{1}{2}\right)\right]
$$

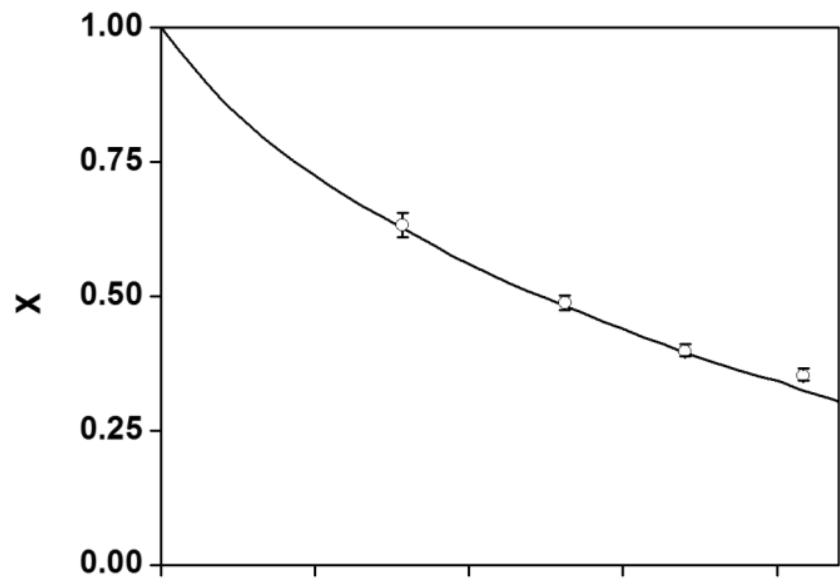

(a)

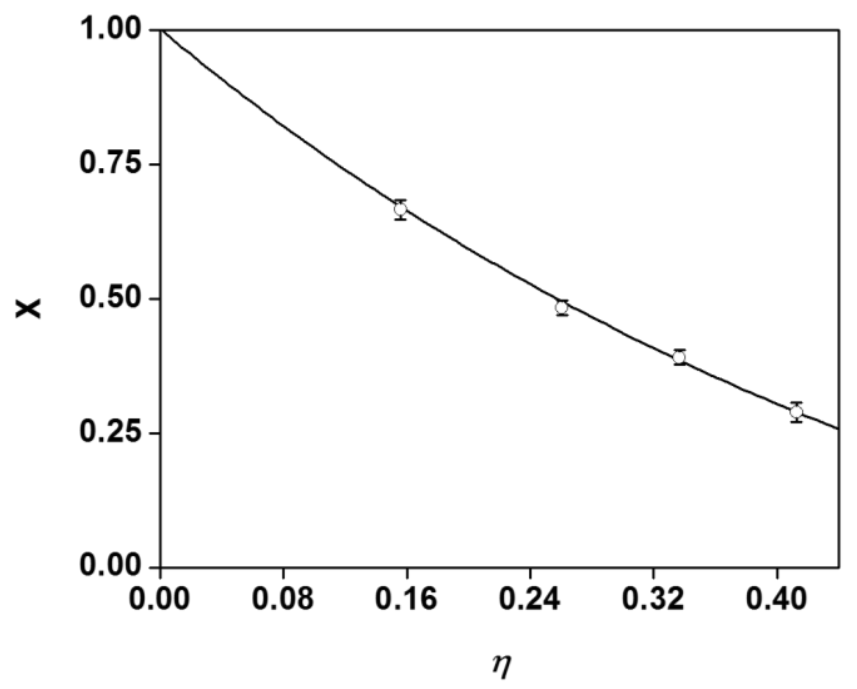

(b)

Figure 8. Mole fraction of monomers, $\mathrm{X}$, in a fluid of molecules having a hard sphere core and off-center square well sites from NPT Monte Carlo simulations (circles) and Wertheim theory (line). Here $\eta=\pi \rho \sigma^{3} / 6$ is reduced density. (a) one bonding site with $\varepsilon_{\mathrm{AB}}=7 k T$; (b) two bonding sites, each with $\varepsilon_{\mathrm{AB}}=5 k T$. Adapted from ref. 117 . 
The Wertheim theory can be extended to molecules having a non-spherical shape, including long chain molecules, by allowing the bonds between some sites to become very strong. For example, if the bonding strength is large but finite, and the fluid consists initially of a mixture of molecules with a single site and others with two bonding sites, chain molecules will form and at equilibrium there will be a distribution of chain lengths, $m$ (the number of mers in each chain). Two versions of the theory for chain molecules exist, the first for strong but finite bonding strengths resulting in a distribution of chain lengths $[119,120]$, and the second for chain molecules whose length is fixed $[114,121]$. For $N_{J}$ chain molecules of fixed length, $m_{J}$, the additional contribution to the compressibility factor from chain formation is

$$
\left.\left.\left(\frac{P}{k T}\right)_{\text {chain }}=\sum_{J=1}^{R} \frac{{ }_{\text {chain }}}{m_{J}} 1\right)\left[1+\left(\frac{\partial \ln }{\partial}\right)_{T}\right)_{T}\right]
$$

where $\underset{\text { chain }}{J}=N_{J} / V$ is the number density of chain molecules and the sum over $J$ is over all chains. Comparisons with molecular simulation have been made for chains of hard sphere [121-123], square well [124] and LJ [125-129] segments. The internal energies are given accurately at moderate to high densities, but the pressures are somewhat too high, particularly for long chains. At low densities the theory is poor because it does not account for the coiling of chains and the resulting intramolecular contributions at these densities.

In the 1980's existing equations of state were for the most part modifications of van der Waals equation, and performed reasonably well for fluids of small nonpolar or slightly polar molecules, but could not describe the effects of molecular association or chain formation. The success of Wertheim's theory in describing the effects of both of these phenomena on the thermodynamic properties led Walter Chapman, Keith Gubbins, George Jackson and Mac Radosz, working at Cornell University and the Exxon Research \& Engineering Company, to propose an equation of state that combined a theory for simple, nonpolar molecules with the Wertheim expressions for association and chain formation $[121,130,131]$. Thus the expression for the residual Helmholtz energy is of the form

$$
A^{\text {res }}=A_{\text {seg }}^{\text {res }}+A_{\text {chain }}^{\text {res }}+A_{\text {assoc }}^{\text {res }}
$$

where the subscripts seg, chain and assoc refer to the contributions from the interaction between chain segments, the formation of the chains and association. They called this 
approach the statistical associating fluid theory (SAFT). Walter Chapman has informed me that the idea for the SAFT equation was initiated during a visit three of the authors made to Kyoto University in March 1987, specifically in a bar to which we had been invited by our host, Professor Hyashi, who had hired a maiko (a geisha in training) to entertain us (Figure 9). In view of the language barrier, the maiko's entertainment consisted of frequent smiling, bowing and sake-pouring, which she did with great enthusiasm. I do not recollect the technical discussion in the bar, although I recall we did discuss the SAFT idea during this trip. However, I do clearly recall that Walter, at the urging of some of the regulars, gave a fine rendition of "I Lost my Heart in San Francisco", one of a small number of karaoke songs in English in the bar's collection. This was received with great enthusiasm by the bar's patrons.

The first term on the right of equation (29) is the Helmholtz energy of a fluid made up of non-bonded chain segments, and can be obtained from one of the theories described earlier for simple non-polar fluids. Frequently used models for the segment term in eqn. (29) have included hard spheres, square well potential [132,133, SAFT-SW], spherical Lennard-Jones [134, 135, SAFT-LJ], a potential of variable range [136, SAFT-VR] and the Mie potential [137-140, SAFT-Mie]. A further variation that gives good agreement with experiment is the use of a chain of hard spheres as the reference system, referred to as 'perturbed-chain SAFT' [141, PC-SAFT]. Group contribution versions of SAFT have also been presented [142,143,144,145, GC-SAFT].

An important early application of SAFT was that of Huang and Radosz [132], who used

as a model for the segment term hard spheres plus an engineering equation of state to describe the dispersion interaction contribution. Their version of SAFT had three adjustable parameters to describe the overlap $(\sigma)$, dispersion $(\varepsilon)$ and chain formation $(m$, the number of chain segments) contributions, plus two parameters, $\varepsilon_{b o n d}$ and $\kappa$ to describe the association. They obtained these parameters from fits to saturated liquid vapor pressures and densities for over one hundred pure compounds and their mixtures and found successful agreement with the experimental data. 


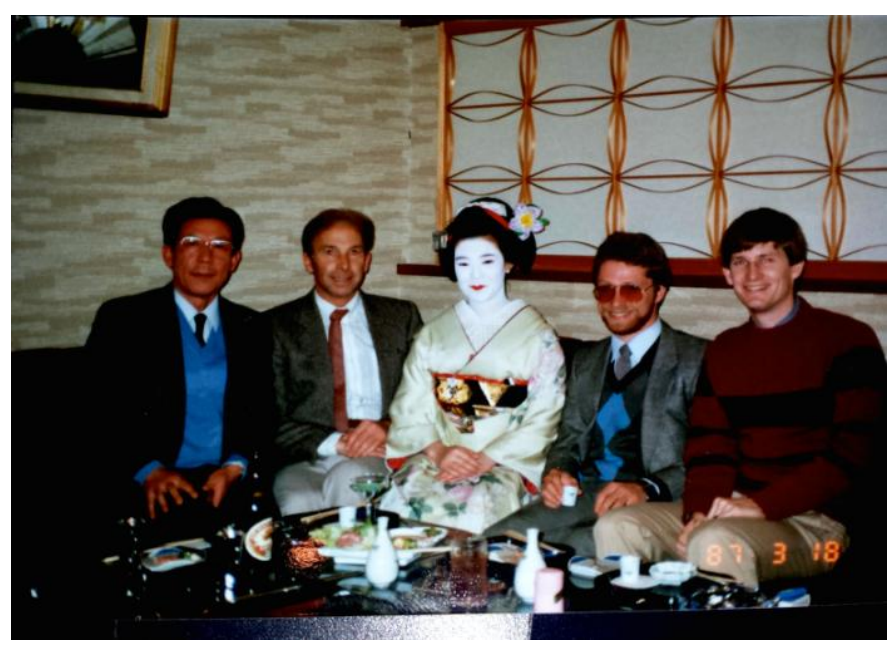

Figure 9. Birth of SAFT? (1. to r.) Professor Hyashi (our host), Keith Gubbins, maiko, George Jackson, Walter Chapman at the bar in Kyoto, 1987.

Equation (23) is the first order thermodynamic perturbation theory (TPT1) version of Wertheim's theory. The first order theory is the simplest version and the one that has been widely used. However because of the neglect of higher order graphs, TPT1 has some limitations. For example, TPT1 does not predict the influence of bond angles on thermodynamic properties, nor does it allow for the formation of ring structures. Extension of the perturbation series to second order yields TPT2, which accounts for higher order graphs with two or more incident bonds, so that the dependence on bond angles is included. The second order term involves an integration over the three-body correlation function for the reference system of segments. Many other extensions of the theory have been proposed in recent years, and these have been reviewed recently $[139,140,146]$.

As an illustration of the ability of the SAFT theory to describe the thermodynamic properties of a particularly challenging liquid, we show in Figures 10 and 11 results for water [139]. The theory is at the TPT1 level using the Mie potential (with the exponent on the dispersion term fixed at 6) for segments, and the water model has 4 off-center square well sites, 2 representing the lone pair electrons (' $\mathrm{e}$ ' sites) and 2 representing the $\mathrm{H}$ atoms ('H' sites). Only 'H-e' interactions between unlike sites are allowed. The calculation involves 5 parameters, the 3 parameters in the Mie potential and 2 (energy and volume) for the bonding sites, which are determined from fits to the vapor-liquid equilibrium data. With the exception of the critical region itself, where the theory does not account for the 
non-analytic behavior, the fit to all properties is very good, including the single-phase properties shown in Figure 11, which were not used in the parameter fitting.
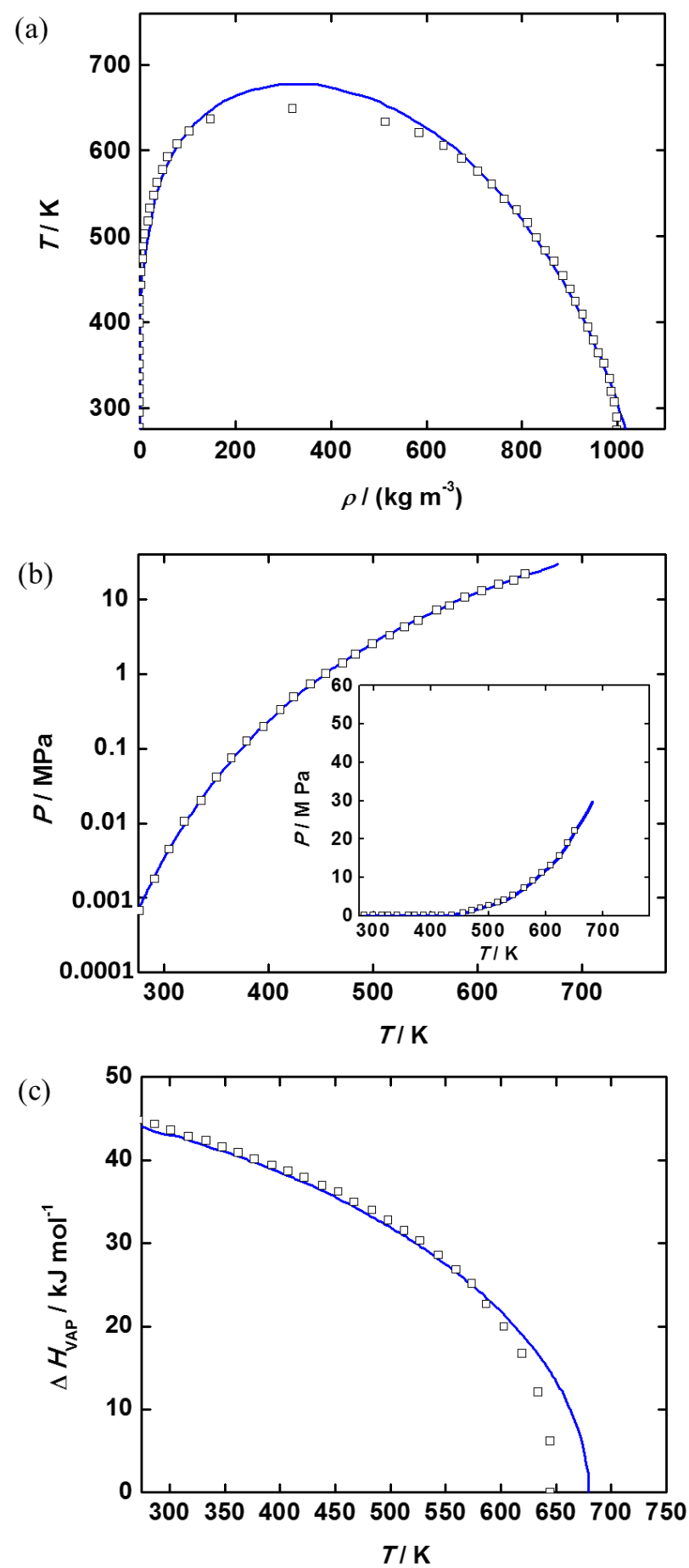
Figure 10. Comparison of SAFT-VR and experimental data for coexistence properties of water: (a) saturated vapor and liquid densities; (b) vapor pressure; (c) enthalpy of vaporization. The symbols are smoothed experimental data from NIST [147], lines are the theory. Adapted from ref. 139.
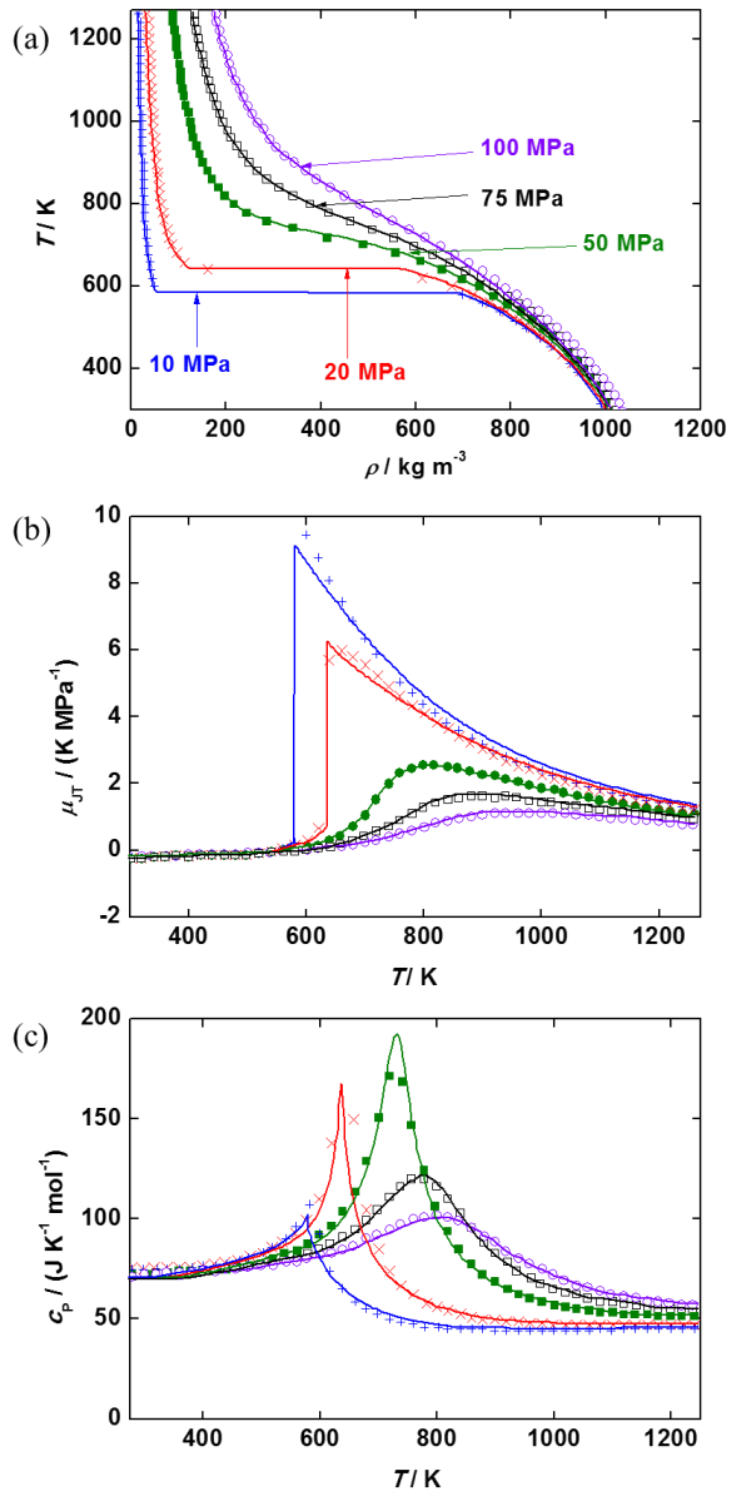

Figure 11. Single phase properties of water from experiment [147] and SAFT-VR theory: (a) density; (b) the Joule-Thomson coefficient; (c) the isobaric heat capacity, at pressures of 10 (blue curves and pluses), 20 (red curves and crosses), 50 (green curves and closed squares), 75 (black curves and open squares) and 100 (purple curves and circles) MPa. Adapted from ref. 139. 
An application of the theory to mixtures is illustrated in Figure 12, which shows vapor-liquid equilibria for the water-methane system. This is a Class III binary mixture in the classification of van Konynenburg and Scott [148], among the most non-ideal mixtures in nature. In this case one mixture parameter, the energy in the Mie potential, was fitted to the data.

(a)

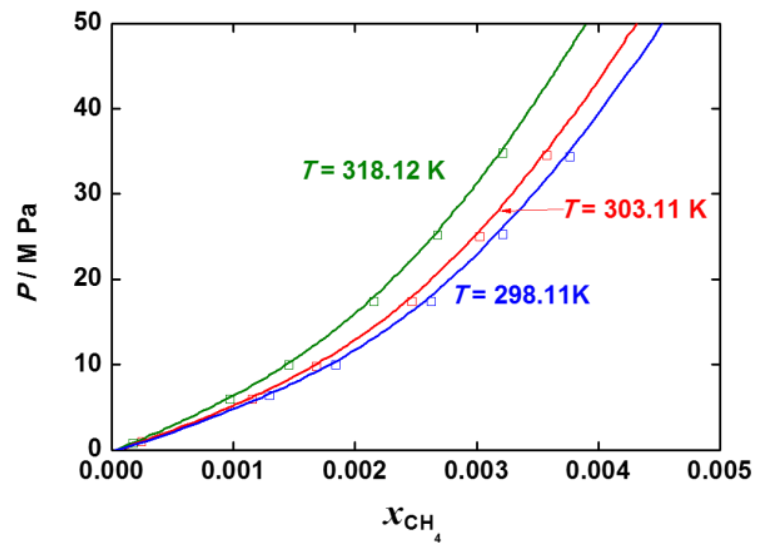

(b)

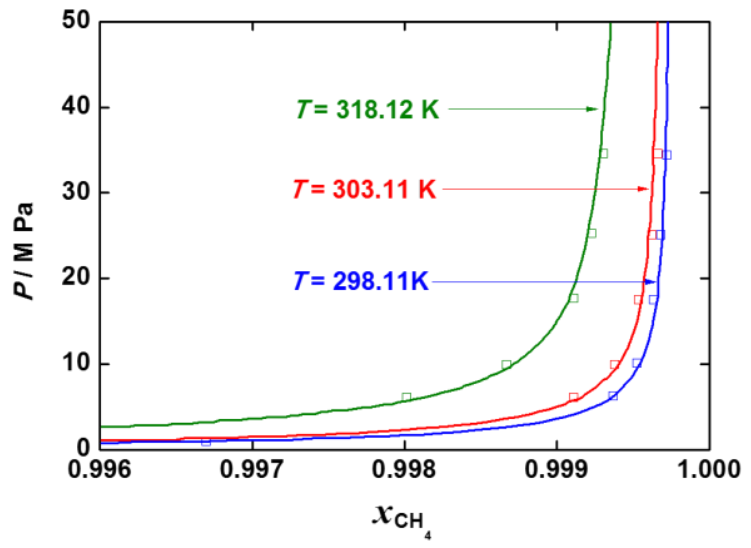

Figure 12. Fluid phase equilibria data for water-methane mixtures at temperatures of 298.11, 303.11 and 318.12 K from experiment (points, 149) and SAFT-VR theory (lines). Adapted from ref. 139.

\section{Conclusions}

Starting with the work of John Barker in 1951 on perturbation theory for polar fluids, there have been steady improvements in our ability to predict the thermodynamic behavior of fluids in which electrostatic forces are important. Such perturbation expansions about a reference fluid of spherical, nonpolar molecules do not converge well for strongly acentric forces, due to the large 
influence of the electrostatic forces on the fluid structure which is not incorporated into the reference system (see, e.g., Fig. 5). However, resummation methods can yield good results, as with the Padé approximant to the Barker series proposed by Stell et al. ${ }^{80}$ The inclusion of effects of induction forces followed a somewhat analogous path. For liquids both the anisotropy of the polarizability and higher many-body contributions led to major difficulties in conventional perturbation theories. Again, resummation methods proposed by Wertheim [86] in his renormalization theory provided a satisfactory solution.

The Wertheim theory TPT1 [110,111] was a major advance in our ability to account for the effects of association. Again, although the theory uses a spherical, nonpolar molecule reference system, a renormalization of the series was able to give excellent agreement with simulation results. In recent years there have been many successful applications to more complex systems, such as polymers, proteins, colloids, interfacial properties, etc. At the present time the successful prediction of thermodynamic properties is limited mainly by our knowledge of the force fields, rather than limitations of the statistical mechanical treatment.

Acknowledgments. I am grateful to George Jackson, Walter Chapman and Amparo Galindo for helpful discussions and comments, and to an anonymous reviewer for reminding me of important work on the influence of induction forces in polar liquids. I also thank Rong ('Ruby') An for help in preparing the manuscript and figures, and Carol Hall for providing the portrait of George Stell. This work was supported in part by NSF grant no. CBET-1160151.

${ }^{1}$ C.G. Gray and K.E. Gubbins, Theory of Molecular Fluids. 1. Fundamentals, Ch. 5, Oxford University Press, Oxford (1984).

2 J.S. Rowlinson, Liquids and Liquid Mixtures, Ch. 8 \& 9, Butterworths Scientific Publications (1959).

3 I.R. McDonald, “Equilibrium Theory of Liquid Mixtures”, in Statistical Mechanics, vol. 1, ed. K. Singer, Specialist Periodical Reports, The Chemical Society, London (1973).

${ }^{4}$ I.R. McDonald, Ann. Reports Chem. Soc. London (1973)

5 W.R. Smith, "Perturbation Theory in Classical Statistical Mechanics of Fluids", in Statistical Mechanics, vol. 1, ed. K. Singer, Specialist Periodical Reports, The Chemical Society, London (1973).

6 J.A. Barker and D. Henderson, "What is liquid - understanding states of matter", Rev. Modern Phys., 48, 587-671 (1976).

7 J.S. Rowlinson, "Van der Waals and the Physics of Liquids", in "J.D. van der Waals: On the Continuity of the Gaseous and Liquid States", ed. J.S. Rowlinson, North-Holland, Amsterdam (1988). 
8 K.E. Gubbins, "The theory of non-electrolyte solutions: an historical review", Mol. Phys., 111, 3666-3697 (2013).

9 J.W. Gibbs, "Elementary Principles of Statistical Mechanics", Yale University Press, New Haven (1902).

${ }^{10}$ L.S. Ornstein, "Applications of Gibbs' statistical mechanics to problems of the molecular theory", doctoral dissertation, University of Leyden (1908).

${ }^{11}$ L.S. Ornstein and F. Zernike, "Accidental deviations of density and opalescence at the critical point of a single substance", Poc. Akad. Sci. (Amsterdam), 17, 793-806 (1914).

12 G.S. Rushbrooke and H.I. Scoins, "On the theory of fluids", Proc. Roy. Soc. A (London), 216, 203-218 (1953).

13 J.M.J. van Leeuwen, J. Groeneveld and J. de Boer, Physica, 25, 792 (1959); M.S. Green, Hughes Aircraft Co. Report (Sept. 1959); M.S. Green, J. Chem. Phys., 33, 1403 (1960);T. Morita and K. Hirioke, Prog. Theoret. Phys., 23, 1003 (1960); E. Meeron, J. Math. Phys., 1, 192 (1960).

${ }^{14}$ J.K. Percus and G.J. Yevick, "Analysis of classical statistical mechanics by means of collective coordinates", Phys. Rev., 110, 1-13 (1958).

${ }^{15}$ M.S. Wertheim, "Exact solution of the Percus-Yevick integral equation for hard spheres", Phys. Rev. Lett., 10, 321-323 (1963).

${ }^{16}$ E. Thiele, "Equation of state for hard spheres", J. Chem. Phys., 39, 474-479 (1963).

17 K.E. Gubbins and J.D. Moore, "Molecular Modeling of Matter: Impact and Prospects in Engineering", Ind. Eng. Chem. Res., 49, 3026-3046 (2010).

18 N. Metropolis, "The Beginning of the Monte Carlo Method", Los Alamos Science, 15, 125 (1987). (Special issue dedicated to Stanislaw Ulam).

19 'Statistical sampling' methods have a much longer history and were used in mathematics and physics applications long before electronic computers became available, but were tedious to use (see 17 and references therein). Metropolis [18] has described how the idea for naming his method the 'Monte Carlo' method came about following conversations with Stan Ulam, who had an uncle who liked to frequent the gaming halls of Monte Carlo. ${ }^{20}$ N. Metropolis, A.W. Rosenbluth, M.N. Rosenbluth, A.H. Teller and E. Teller, "Equation of state calculations by fast computing machines", J. Chem. Phys., 21, 1087-1092 (1953). ${ }^{21}$ M.N. Rosenbluth and A.W. Rosenbluth, "Further results on Monte-Carlo equations of state", J. Chem. Phys., 22, 881-884 (1954).

22 B.J. Alder and T.E. Wainwright, in International Symposium on Statistical Mechanical Theory of Transport Processes, Brussels, 1956, ed. I. Prigogine, Interscience, New York (1958).

${ }^{23}$ B.J. Alder and T.E. Wainwright, "Phase Transition for a Hard Sphere System", J. Chem. Phys., 27, 1208 (1957).

24 B.J. Alder and T.E. Wainwright, "Studies in Molecular Dynamics", J. Chem. Phys., 31, 459 (1959).

${ }^{25}$ W.W. Wood, "Early History of Computer Simulation in Statistical Mechanics", in Molecular Dynamics Simulation of Statistical Mechanical Systems, ed. G. Ciccotti and W.G. Hoover, Proc. Int. School of Physics 'Enrico Fermi', Course 97, p.3, North-Holland, Amsterdam (1986).

${ }^{26}$ N.F. Carnahan and K.E. Starling, "Equation of state for nonattracting hard spheres", J. Chem. Phys., 51, 635-636 (1969). 
${ }^{27}$ T. Boublik, "Hard sphere equation of state", J. Chem. Phys., 53, 471-472 (1970).

${ }^{28}$ G.A. Mansoori, N.F. Carnahan, K.E. Starling and T.W. Leland, "Equilibrium thermodynamic properties of mixtures of hard spheres", J. Chem. Phys., 54, 1523-1525 (1971).

${ }^{29}$ The HNC theory does, however, give quite good results for the restricted primitive model of ionic solutions, in which the electrolyte solution is treated as made up of hard sphere anions and cations having the same diameter but charges of opposite sign, immersed in a continuum solvent. See, for example, ref. 30 and also: J.C. Rasaiah, D.N. Card and J.P. Valleau, J. Chem. Phys., 56, 248 (1972).

${ }^{30}$ For a review of these theories and comparison with simulation and experimental results up to 1972 see: R.O. Watts, "Integral equation approximations in the theory of fluids", in Specialist Periodical Report: Statistical Mechanics, Vol. 1, ed. K. Singer, p.1, The Chemical Society, London (1983).

31 The theory was first presented by Longuet-Higgins at a conference in Manchester in October 1950; a report of the meeting is given in H.C. Longuet-Higgins, Nature, 167, 12 (1951). The theory is published in: H.C. Longuet-Higgins, Proc. Roy. Soc. London, A205, 247 (1951).

32 T.W. Leland, Jr., P.S. Chappelear and B.W. Gamson, "Prediction of vapor-liquid equilibria from the corresponding states principle”, Amer. Inst. Chem. Engr. J., 8, 482-489 (1962). ${ }_{33}$ A.M. Georgoulaki, I.V. Ntouros, D.P. Tassios and A.V. Panagiotopoulos, "Lennard-Jones mixtures - simulation and van-der-Waals 1-fluid theory", Fluid Phase Eqba., 100, 153-170 (1994).

34 T.W. Leland, J.S. Rowlinson and G.A. Sather, "Statistical thermodynamics of mixtures of molecules of different sizes", Trans. Faraday Soc., 546, 1447-1460 (1968).

35 J.D. van der Waals, Zeit. Phys. Chem., 5, 1331 (1890); Arch. Néerl., 24, 1 (1891).

36 R.W. Zwanzig, "High temperature equation of state by a perturbation method. 1. Nonpolar gases", J. Chem. Phys. 22, 1420 (1954).

37 J.S. Rowlinson, "Statistical mechanics of systems with steep intermolecular potentials", Mol. Phys., 8, 107-115 (1964).

38 J.A. Barker and D. Henderson, Disc. Faraday Soc., 43, 50 (1967).

39 J.A. Barker and D. Henderson, "Perturbation theory and equation of state for fluids. II. A successful theory of liquids", J. Chem. Phys., 47, 4714 (1967).

${ }^{40}$ J.D. Weeks and D. Chandler, "Equilibrium structure of simple liquids", Phys. Rev. Lett. 25, 149-152 (1970); J.D. Weeks, D. Chandler and H.C. Andersen, "Role of repulsive forces in determining equilibrium structure of simple liquids", J. Chem. Phys., 54, 5237-5247 (1971). See also: K.E. Gubbins, W.R. Smith, M.K. Tham, and E.W. Tiepel, "Perturbation Theory for the Radial Distribution Function," Molecular Physics, 22, 1089 (1971).

${ }^{41}$ Ref. 1, Figures 4.2 to 4.4 .

42 J.D. van der Waals, Verhand. Kon. Akad. Weten. Amsterdam, 21, No. 5 (1881); Ann. Physik Beibl., 5, 27 (1881).

43 Z. Wreblewski, Sitzungsber. Kais. Akad. Wiss. Wien, Sekt. IIa, 97, 1321 (1888).

44 J.S. Rowlinson, "Sir James Dewar, 1842-1923. A Ruthless Chemist" Ashgate Pub. Co., Burlington (2012).

45 J. Dewar, Presidential Address, Rep. British Association, 72, 3-50, see p. 29 (1902).

46 J.S. Rowlinson, "Cohesion. A Scientific History of Intermolecular Forces", p. 186, Cambridge University Press, Cambridge (2002). 
47 H. Kammerlingh Onnes, "Algemeene theorie der vloeistoffen", Verhand. Konink. Akad. Weten. Amstedam, 21, in three parts, Nos. 4, 5, 6 (1880-1881).

48 Ref. 7, p. 23.

${ }^{49}$ J. Levelt Sengers, "How Fluids Unmix. Discoveries by the School of Van der Waals and Kammerlingh Onnes", Chapter 3, Koninklijke Nederlandse Akademie van Wetenschappen, Amsterdam (2002).

50 J. de Boer and A. Michels, "Contribution to the quantum mechanical theory of the equation of state and the law of corresponding states. Determination of the law of force for helium”, Physica, 5, 945 (1938).

51 K.S. Pitzer, “Corresponding states for perfect liquids”, J. Chem. Phys., 7, 583 (1939).

52 T.M. Reed and K.E. Gubbins, Applied Statistical Mechanics, Ch. 11, McGraw-Hill, New York (1973).

53 J.A. Barker, "Conformal solution theory and dipole interaction", J. Chem. Phys., 19, 1430 (1951).

54 J.A. Barker, "Statistical mechanics of interacting dipoles", Proc. Roy. Soc. London, A219, 367 (1953).

55 J.A. Pople, "Molecular association in liquids. III. A theory of cohesion of polar liquids", Proc. Roy. Soc. London, A215, 67 (1952).

56 J.A. Pople, "The statistical mechanics of systems with non-central force fields", Disc. Faraday Soc., 15, 35 (1953).

57 J.A. Pople, "The statistical mechanics of assemblies of axially symmetric molecules. I. General theory", Proc. Roy. Soc. London, A211, 498 (1954).

58 J.A. Pople, "The statistical mechanics of assemblies of axially symmetric molecules. II. Second virial coefficients", Proc. Roy. Soc. London, A211, 508 (1954).

59 J.E. Lennard-Jones and A.F. Devonshire, "Critical Phenomena in Gases - I" Proc. Roy. Soc. London, A163, 53 (1937).

60 H. Eyring and J.O. Hirschfelder, "The Theory of the Liquid State", J. Phys. Chem., 41, 249 (1937).

61 R. Fowler and E.A. Guggenheim, Statistical Thermodynamics, p. 322, Cambridge

University Press, Cambridge (1949).

62 E.A. Guggenheim, Mixtures. The Theory of the Equilibrium Properties of Some Simple Classes of Mixtures, Solutions and Alloys, Oxford University Press, Oxford (1952).

${ }^{63}$ E.B. Smith and K.R. Lea, "A Monte-Carlo equation of state for mixtures of hard-sphere molecules", Nature, 186, 174-174 (1960).

64 E.B. Smith and K.R. Lea, "Thermodynamic properties of mixtures of hard sphere molecules by Monte Carlo methods", Trans. Faraday Soc., 59, 1535-1543 (1963).

65 D. Cook and J.S. Rowlinson, "Deviations from the principle of corresponding states", Proc. Roy. Soc. London, A219, 405 (1953).

66 Ref. 1, Sec. 4.9.

67 J.S. Rowlinson and J.R. Sutton, "The statistical thermodynamics of solutions of nonspherical molecules. I. The thermodynamic properties", Proc. Roy. Soc. London, A229, 271 (1955).

68 K.E. Gubbins and C.G. Gray, "Perturbation theory for the angular pair correlation function in molecular fluids”, Molec. Phys., 187 (1972). 
${ }^{69}$ G. Stell, J.C. Rasaiah and H. Narang, "Thermodynamic perturbation theory for simple polar fluids, I", Molec. Phys., 23, 393 (1972).

70 S.S. Wang, P.A. Egelstaff, C.G. Gray and K.E. Gubbins, "Monte Carlo study of the angular pair correlation function in a liquid with quadrupolar forces", Chem. Phys. Lett., 24, 453 (1974).

71 G.N. Patey and J.P. Valleau, "Free-energy of spheres with dipoles - Monte-Carlo with multistage sampling", Chem. Phys. Lett., 21, 297-300 (1973); "Dipolar hard spheres Monte-Carlo study", J. Chem. Phys., 61, 534-540 (1974).

72 L. Verlet and J.J. Weis, "Perturbation theories for polar fluids", Molec. Phys., 28, 665-682 (1974).

73 I.R. McDonald, "Application of thermodynamic perturbation-theory to polar and polarizable fluids", J. Phys., C7, 1225-1236 (1974).

74 G.N. Patey and J.P. Valleau, "Fluids of spheres containing quadrupoles and dipoles - study using perturbation-theory and Monte-Carlo computations", J. Chem. Phys., 64, 170-184 (1976).

75 K.E. Gubbins and C.H. Twu, "Thermodynamics of polyatomic fluid mixtures. I. Theory", Chem. Eng. Sci., 33, 863-878 (1978).

76 D.E. Sullivan, J.M. Deutch and G. Stell, “Thermodynamics of polar lattices”, Mol. Phys., 28, 1359-1371 (1974).

77 S. Jiang and K.S. Pitzer, "Thermodynamic properties of mixtures of dipolar and quadrupolar hard spheres: theory and simulation", J. Chem. Phys., 102, 7632 (1995).

78 H. Narang, M.Sc. thesis, State University of New York at Stony Brook (1972).

${ }^{79}$ G.S. Rushbrooke, G. Stell and J.S. Høye, "Theory of polar liquids. I. Dipolar hard spheres", 26, 1199 (1973).

80 G. Stell, J.C. Rasiah and H. Narang, "Thermodynamic perturbation theory for simple polar fluids. II", Mol. Phys., 27, 1393 (1974).

81 B. Moser, K. Lucas and K.E. Gubbins, "Predictive methods for fluid phase-equilibria molecular theory and empirical equations of state", Fluid Phase Eqba., 7, 153-179 (1981).

82 K.O. Shukla, B. Moser and K. Lucas, "Molecular thermodynamics on the basis of an equation of state for argon. 2. Binary-mixtures of weakly nonspherical molecules", Fluid Phase Eqba., 17, 19-55 (1984).

83 C.G. Gray, K.E. Gubbins and C.G. Joslin, Theory of Molecular Fluids. 2. Applications, Secs. 6.11, 7.8, Oxford University Press, Oxford (2011).

84 B. Larsen, J.C. Rasaiah and G. Stell, "Thermodynamic perturbation-theory for multipolar and ionic liquids", Molec. Phys., 33, 987-1027 (1977).

85 G.N. Patey, G.M. Torrie and J.P. Valleau, "A Monte Carlo study of the dense polarizable dipolar hard sphere fluid", J. Chem. Phys., 71, 96-103 (1979).

86 M.S. Wertheim, "Theory of polar fluids. V. Thermodynamics and thermodynamic perturbation theory", Molec. Phys., 37, 83-94 (1979).

87 S.L. Carnie and G.N. Patey, "Fluids of polarizable hard spheres with dipoles and tetrahedral quadrupoles. Integral equation results with application to liquid water", Molec. Phys., 47, 1129-1151 (1982).

88 M. Kleiner and J. Gross, "An equation of state contribution for polar components: polarizable dipoles", Amer. Inst. Chem. Eng. J., 52, 1951-1961; see also C.G. Gray and K.E. Gubbins, ref. 1, Sec. 4.10. 
${ }^{89}$ V. Venkatasubramanian, Ph.D. Thesis, Cornell University (1983); V. Venkatasubramanian, K.E. Gubbins, C.G. Gray and C.G. Joslin, "Induction effects in polar-polarizable liquid mixtures. Calculations of thermodynamic properties using renormalized perturbation theory", Molec. Phys., 52, 1411-1429 (1984).

90 C.G. Gray, C.G. Joslin, V. Venkatasubramanian and K.E. Gubbins, "Induction effects in fluid mixtures of dipolar-quadrupolar polarizable molecules”, Molec. Phys., 54, 1129-1148 (1985).

91 C.G. Joslin, C.G. Gray and K.E. Gubbins, "Renormalized perturbation theory for dipolar and quadrupolar polarizable liquids", Molec. Phys., 54, 1117-1128 (1985).

92 W.G. Chapman, K.E. Gubbins, C.G. Joslin and C.G. Gray, "Mixtures of polar and associating molecules", Pure Appl. Chem., 59, 53-60 (1987).

93 L.Q. Lobo, L.A.K. Staveley, V. Venkatasubramanian, P. Clancy, K.E. Gubbins, C.G. Gray and C.G. Joslin, "Thermodynamic properties of liquid mixtures of hydrogen chloride and tetrafluoromethane", Fluid Phase Eqba., 22, 89-105 (1985).

94 F.Z. Dolezalek, "Zur theorie der binären gemische und koncentrierte lösungen", Zeit. Phys. Chem., 64, 727 (1908).

95 Such treatments are described well in: J.M. Prausnitz, R.N. Lichtenthaler and E. Gomes de Azevedo, Molecular thermodynamics of fluid-phase equilibria, $3^{\text {rd }}$ edn., Ch. 7, Prentice-Hall, Englewood Cliffs (1999).

96 J.J. van Laar, “Zur theorie der dampfspannungen von binären gemischen”, Zeit. Physik. Chem., 83, 599 (1913).

${ }^{97}$ A good account of the polemic between van Laar and Dolezalek over the 'chemical' theory is given in: E.P. van Emmerik, Dr. J.J. van Laar and the Dutch School of Thermodynamics, Dienst Reprografie, Vrie Universiteit, Amsterdam (2005).

98 J.H. Hildebrand, Solubility, p. 83, The Chemical Catalog Co., New York (1924); also J.H. Hildebrand and R.L. Scott, The solubility of non-electrolytes, $3^{\text {rd }}$ edn., p. 175, Reinhold Pub. Co., New York (1950).

${ }^{99} \mathrm{G}$. Scatchard, "Equilibria in non-electrolyte solutions in relation to the vapor pressures and densities of the components", Chem. Rev., 8321 (1931).

100 E.A. Guggenheim, Mixtures, Clarendon Press, Oxford (1952).

101 J.A. Barker and W. Fock, "Theory of Upper and Lower Critical Solution Temperatures", Disc. Faraday Soc., 15, 188 (1953).

102 J.C. Wheeler, "Exactly soluble 2-component lattice solution with upper and lower critical solution temperatures", J. Chem. Phys., 62, 433 (1975); "Decorated lattice-gas models of critical phenomena in fluids and fluid mixtures", Ann. Rev. Phys. Chem., 28, 411 (1977).

103 J.S. Walker and C.A. Vause, "Theory of closed-loop phase diagrams in binary fluid mixtures", Phys. Lett. A, 79, 421-424 (1980).

104 H.C. Andersen, "Cluster expansions for hydrogen-bonded fluids. 1. Molecular association in dilute gases”, J. Chem. Phys., 59, 4714-4725 (1973); "Cluster expansions for hydrogenbonded fluids. 2. Dense liquids", ibid., 61, 4985-4992 (1974).

105 L.W. Dahl and H.C. Andersen, "Cluster expansions for hydrogen-bonded fluids. 3. Water", J. Chem. Phys., 78, 1962-1979; "A theory of the anomalous thermodynamic properties of liquid water", ibid., 78, 1980-1993 (1983).

106 D. Chandler and L.R. Pratt, "Statistical-mechanics of chemical equilibria and intramolecular structures of nonrigid molecules in condensed phases", J. Chem. Phys., 65, 
2925-2940 (1976); L.R. Pratt and D. Chandler, "Interaction site cluster series for Helmholtz free-energy and variational principle for chemical equilibria and intramolecular structures”, J. Chem. Phys., 66, 147-151 (1977).

107 J.S. Høye and K. Olaussen, "Statistical mechanical model with chemical reaction", Physica $A, \mathbf{1 0 4}, 435-446$ (1980).

108 G.N. Patey and S.L. Carnie, "Theoretical results for aqueous electrolytes - ion-ion potentials of mean force and the solute-dependent dielectric-constant", J. Chem. Phys., 78, 5183-5190 (1983).

109 P.T. Cummings and G. Stell, "Statistical mechanical models of chemical-reactions. Analytic solution of models of A+B reversible AB in the Percus-Yevick approximation", Mol. Phys., 51, 253-287 (1984).

110 M.S. Wertheim, "Fluids with highly directional attractive forces. I. Statistical thermodynamics", J. Stat. Phys., 35, 19 (1984).

111 M.S. Wertheim, "Fluids with highly directional attractive forces. II. Thermodynamic perturbation theory and integral equations", J. Stat. Phys., 35, 35 (1984).

112 C.G. Joslin, C.G. Gray, W.G. Chapman and K.E. Gubbins, "Theory and simulation of associating liquid mixtures. II", Mol. Phys., 62, 843 (1987); see also A. Galindo, S.J. Burton, G. Jackson, D.P. Visco Jr. and D.A. Kofke, "Improved models for the phase behavior of hydrogen fluoride: chain and ring aggregates in the SAFT approach and the AEOS model", Mol. Phys., 100, 2241-2259 (2002); J-P. Hansen and I.R. McDonald, Theory of simple liquids, $4^{\text {th }}$ edn., Sec. 11.10, Academic Press, Oxford (2013); C.G. Gray, K.E. Gubbins and C.G. Joslin, ref. 83, Secs. 6.13 and 7.9.

113 M.S. Wertheim, "Fluids with highly directional attractive forces. III. Multiple attraction sites", J. Stat. Phys., 42, 459 (1986).

114 W.G. Chapman, Ph.D. Thesis, Cornell University (1988). Equations (23) and (24) were derived by Chapman, and are somewhat more general than the equations given in Wertheim's paper. See also ref. 121.

115 W.G. Chapman, K.E. Gubbins, C.G. Joslin and C.G. Gray, "Theory and simulation of associating liquid mixtures", Fluid Phase Eqba., 29, 337 (1986).

116 J. Kolafa and I. Nezbeda, "Monte Carlo simulations on primitive models of water and methanol", Mol. Phys., 61, 161 (1987).

117 G. Jackson, W.G. Chapman and K.E. Gubbins, "Phase equilibria of associating fluids. Spherical molecules with multiple bonding sites”, Mol. Phys., 65, 1 (1988).

118 J.K. Johnson and K.E. Gubbins, "Phase equilibria for associating Lennard-Jones fluids from theory and simulation", Mol. Phys., 77, 1033 (1992).

119 M.S. Wertheim, "Fluids with highly directional attractive forces. IV. Equilibrium polymerization”, J. Stat. Phys., 42, 477 (1986).

120 M.S. Wertheim, "Thermodynamic perturbation theory of polymerization”, J. Chem. Phys., 87, 7323 (1987).

121 W.G. Chapman, G. Jackson and K.E. Gubbins, "Phase equilibria of associating fluids. Chain molecules with multiple bonding sites", Mol. Phys., 65, 1057 (1988).

122 D. Ghonasgi and W.G. Chapman, "Theory and simulation for associating hard chain fluids", Mol. Phys., 83, 145-158 (1994).

123 E.A. Müller and K.E. Gubbins, "Triplet correlation function for hard-sphere systems", Mol. Phys., 80, 91-101 (1993). 
124 M. Banaszak, Y.C. Chien and M. Radosz, "Thermodynamic perturbation-theory - sticky chains and square-well chains", Phys. Rev. E, 48, 3760-3765 (1993); Banaszak, M., Chen, C.K. and Radosz, M., "Copolymer SAFT equation of state. Thermodynamic perturbation theory extended to heterobonded chains", Macromolecules, 29, 6481-6486 (1996); H. Adidharma and M. Radosz, "Prototype of an engineering equation of state for heterosegmented polymers", Ind. Eng. Chem. Res., 37, 4453-4462 (1998).

125 D. Ghonasgi, M.L. Llano-Restrepo and W.G. Chapman, "Henry law constant for diatomic and polyatomic Lennard-Jones molecules”, J. Chem. Phys., 98, 5662-5667 (1993).

126 J.K. Johnson, E.A. Müller and K.E. Gubbins, "Equation of state for Lennard-Jones chains", J. Phys. Chem., 98, 6413-6419 (1994).

127 M. Banaszak, Y.C. Chiew, R. O’Lenick and M. Radosz, “Thermodynamic perturbationtheory - Lennard-Jones chains", J. Chem. Phys., 100, 3803-3807 (1994).

128 D. Ghonasgi and W.G. Chapman, "Prediction of the properties of model polymersolutions and blends", AIChEJ, 40, 878-887 (1994).

129 F.J. Blas and L.F. Vega, "Thermodynamic behavior of homonuclear and heteronuclear Lennard-Jones chains with association sites from simulation and theory", Mol. Phys., 92, 135-150 (1997).

130 W.G. Chapman, K.E. Gubbins, G. Jackson and M. Radosz, "SAFT: Equation-of-state solution model for associating fluids", Fluid Phase Eqba., 52, 31 (1989).

131 W.G. Chapman, K.E. Gubbins, G. Jackson and M. Radosz, "New reference equation of state for associating fluids", Ind. Eng. Chem. Res., 29, 1709 (1990).

132 S.H. Huang and M. Radosz, "Equation of state for small, large, polydisperse and associating molecules”, Ind. Eng. Chem. Res., 29, 2284 (1990); S.H. Huang and M. Radosz, "Equation of state for small, large, polydisperse and associating molecules - extension to fluid mixfures", Ind. Eng. Chem. Res., 30, 1994 (1991).

133 Y.H. Fu and S.I. Sandler, "A simplified SAFT equation of state for associating compounds and mixtures", Ind. Eng. Chem. Res. 34, 1897 (1995).

134 T. Kraska and K.E. Gubbins, "Phase Equilibria Calculations with a Modified SAFT Equation of State: I. Pure Alkanes, Alkanols and Water", Ind. Eng. Chem. Res., 35, 4727 (1996); T. Kraska and K.E. Gubbins, "Phase Equilibria Calculations with a Modified SAFT Equation of State: II. Binary Mixtures of n-Alkanes, 1-Alkanols and Water", Ind. Eng. Chem. Res., 35, 4738 (1996). 135 F. Blas and L.F. Vega, "Prediction of binary and ternary diagrams using the statistical associating fluid theory (SAFT) equation of state", Ind. Eng. Chem. Res., 37, 660 (1998). 136 A. Gil-Villegas, A. Galindo, P.J. Whitehead, S.J. Mills, G. Jackson and A.N. Burgess, "Statistical associating fluid theory for chain molecules with attractive potentials of variable range", J. Chem. Phys., 106, 4168 (1997); A. Galindo, L.A. Davies, A. Gil-Villegas and G. Jackson, "The thermodynamics of mixtures and the corresponding mixing rules in the SAFT-VR approach for potentials of variable range”, Mol. Phys., 93, 241 (1998).

137 T. Lafitte, A. Apostolakou, C. Avendaño, A. Galindo, C.S. Adjiman, E.A. Müller and G. Jackson, "Accurate statistical associating fluid theory for chain molecules composed of Mie segments", J. Chem. Phys., 139, 154504 (2013).

138 V. Papaioannou, T. Lafitte, C. Avendaño, C.S. Adjiman, G. Jackson, E.A. Müller and A. Galindo "Group contribution methodology based on the statistical associating fluid theory for heteronuclear molecules formed from Mie segments", J. Chem. Phys., 140, 054107 (2014). 
139 S. Dufal, T. Lafitte, A.J. Haslam, A. Galindo, G.N.I. Clark, C.Vega and G. Jackson, "The A in SAFT: developing the contribution of association to the Helmholtz free energy within a Wertheim TPT1 treatment of generic Mie fluids", Mol. Phys., 113, 948-984 (2015). 140 S. Dufal, T. Lafitte, A. Galindo, G. Jackson and A.J. Haslam "Developing intermolecularpotential models for use with the SAFT-VR Mie equation of state, AIChEJ, 61, 2891 (2015). 141 J. Gross and G.Sadowski, "Perturbed-chain SAFT: an equation of state based on a perturbation theory for chain molecules", Ind. Eng. Chem. Res., 40, 1244 (2001). 142 S. Tamouza, J.-P. Passarello, P. Tobaly and J.-C. de Hemptinne, "Group contribution method with SAFT equation of state applied to vapor-liquid equilibria of various hydrocarbon series", Fluid Phase Eqba., 222-223, 67 (2004).

143 A. Lymperiadis, C.S. Adjiman, G. Jackson and A. Galindo, "A group contribution method for associating chain molecules based on the statistical associating fluid theory (SAFTgamma)", J. Chem. Phys., 127, 234903 (2007).

144 A. Lymperiadis, C.S. Adjiman, A. Galindo and G. Jackson, "A generalization of the SAFTgemma group contribution method for groups comprising multiple spherical segments", Fluid Phase Eqba., 274, 85 (2008).

145 Y. Peng, K.D. Goff, M.C. dos Ramos and C. McCabe, "Developing a predictive groupcontribution-based SAFT-VR equation of state", Fluid Phase Eqba., 277, 131 (2009).

146 Ref. 83, Sec. 6.13.

147 P. Linstrom and W. Mallard, eds., NIST Chemistry Web-Book, NIST Standard Reference Database Number 69, National Institute of Standards and Technology, Gaithersburg, MD (2013).

148 P.H. van Konynenburg and R.L. Scott, "Critical lines and phase equilibria in binary van der Waals mixtures", Phil. Trans. Roy. Soc. London, 298, 495 (1980).

149 A. Chapoy, C. Coquelet and D. Richon, "Solubility measurements and modeling of water in the gas phase of the methane/water binary system at temperatures from 283.08 to 318.12 K and pressures up to 34.5 MPa", Fluid Phase Equilib., 214, 101 (2003). 